\title{
Helminth parasites of freshwater fish from Central America
}

\author{
GUILLERMO SALGADO-MALDONADO
}

Universidad Nacional Autónoma de México, Instituto de Biología, Laboratorio de Helmintología, Apartado Postal 70-153 CP 04510, México D. F. Mexico.E-mail: gsalgado@ibiologia.unam.mx

\begin{abstract}
This study is a compilation of current knowledge of the taxonomic composition and distribution of the helminth parasites of freshwater fish in Central America. A list of 111 adult helminth species up to day reported from 17 freshwater fish families from Central America was compiled. The data show a helminth parasite fauna in freshwater fish that is typical to the region. One hundred and two of the known helminth species are endemic to the area, 32 of which can be derived from South American genera. Nematodes were the most abundant group, followed by monogeneans and trematodes. None of the 29 helminth families recorded to date is exclusive to Central America, while 16 of the 65 recorded genera have been only recorded from this area. Twenty three of these genera are South American lineages. The data suggest that helminth parasites of freshwater fish from Central America constitute a recent fauna derived mainly from South America but not found there.
\end{abstract}

Key words: parasites; helminths; checklist; freshwater fishes; Central America; zoogeography; Platyhelminthes; Trematoda; Monogenea; Cestoda; Nematoda; Acanthocephala; Cichlidae; Poeciliidae; Characidae; Heptapteridae; cichlids; poeciliids endemism.

\section{Introduction}

Freshwater fish in Central America constitute a faunal assemblage distinct from those of North and South America, and the species within it are parasitized by their own helminth fauna. Between the Isthmus of Tehuantepec, in southern Mexico, and the Isthmus of Panama there are only two suckers and one catfish species, and no records of minnows, perches, darters or sunfishes, which jointly constitute the characteristic fauna of North America. With the exception of Lepisosteidae, no Nearctic fish families have been able to establish themselves farther south than Guatemala. Similarly, most South American fish families are not distributed further north than the Isthmus of Panama. Some of these families extend into Costa Rica, and only a few characids are found in Guatemala and southern Mexico; the siluriforms and characids are characteristic South American elements that are not widely distributed in Central America (Miller 1966; Myers 1966; Bussing 1985, 1998).

Many helminths parasitizing Central America fish are endemic to the region (Salgado-Maldonado 2006). But in general the parasites of freshwater fishes in Central America are poorly known (Choudhury et al. 2002; Scholz et al. 2004). In response to this, the present study is a compilation of current knowledge of the taxonomic composition and distribution of the helminth parasites of freshwater fish in Central America. Species lists are important because they are needed to successfully document and understand the causes and consequences of biotic diversity. Species lists form a vital element of distribution studies because they aid in generating hypotheses to guide the application of experimental or comparative methods. More research is needed especially in the tropical biodiversity hotspots, identification resources, including field guides, monographs 
and species lists are required. Species lists improve the ease with which taxonomic information can be accessed. A list provides a portal to the information available about different species. They can be used to collate the literature and specimen information required to do taxonomy. Lists made available current nomenclature and specimens occurrence records. It is expected that this checklist will facilitate future research on taxonomy, biogeography, ecology, and biodiversity.

In this study I examine helminth parasite taxonomic characteristics and geographic distribution to describe this fauna's apparent geographic sources. The fundamental postulate underlying this research is that each host family has a typical set of helminth parasites and the distribution of these helminths reflects that of the fish families they parasitize (Salgado-Maldonado et al. 2005; Salgado-Maldonado 2006; Aguilar-Aguilar et al. 2008). This pattern has been empirically recognized by researchers studying the helminths of Central American fish (Scholz \& Salgado-Maldonado 1994; Scholz et al. 1995b, 1996c; Mendoza-Franco et al. 2000; Vidal-Martínez et al. 2001b; Kritsky \& Mendoza-Franco, 2003). Dogiel (1961), Chubb (1963), and Wootten (1973) all acknowledged the close relationship between parasites and their hosts, and between hosts and their environment, as determinants of the character of a regional helminth fauna. This pattern was empirically recognized also from several freshwater drainage basins in Mexico (Salgado-Maldonado et al. 2001, 2004a, b). Despite that these ideas have been established for a long time, Pérez \& Choudhury (2005) mistakenly identified a large number of endemisms among the helminth parasites of freshwater fish in Mexico; political boundaries do not necessarily delimit biological distribution. In contrast Vidal-Martínez \& Kennedy (2000) proposed that portions of Neotropical Mexico particularly the southeast, form part of a Mesoamerican region, which is supported by the data and analysis of Aguilar-Aguilar et al. (2003) and Salgado-Maldonado (2006), as well as the present study.

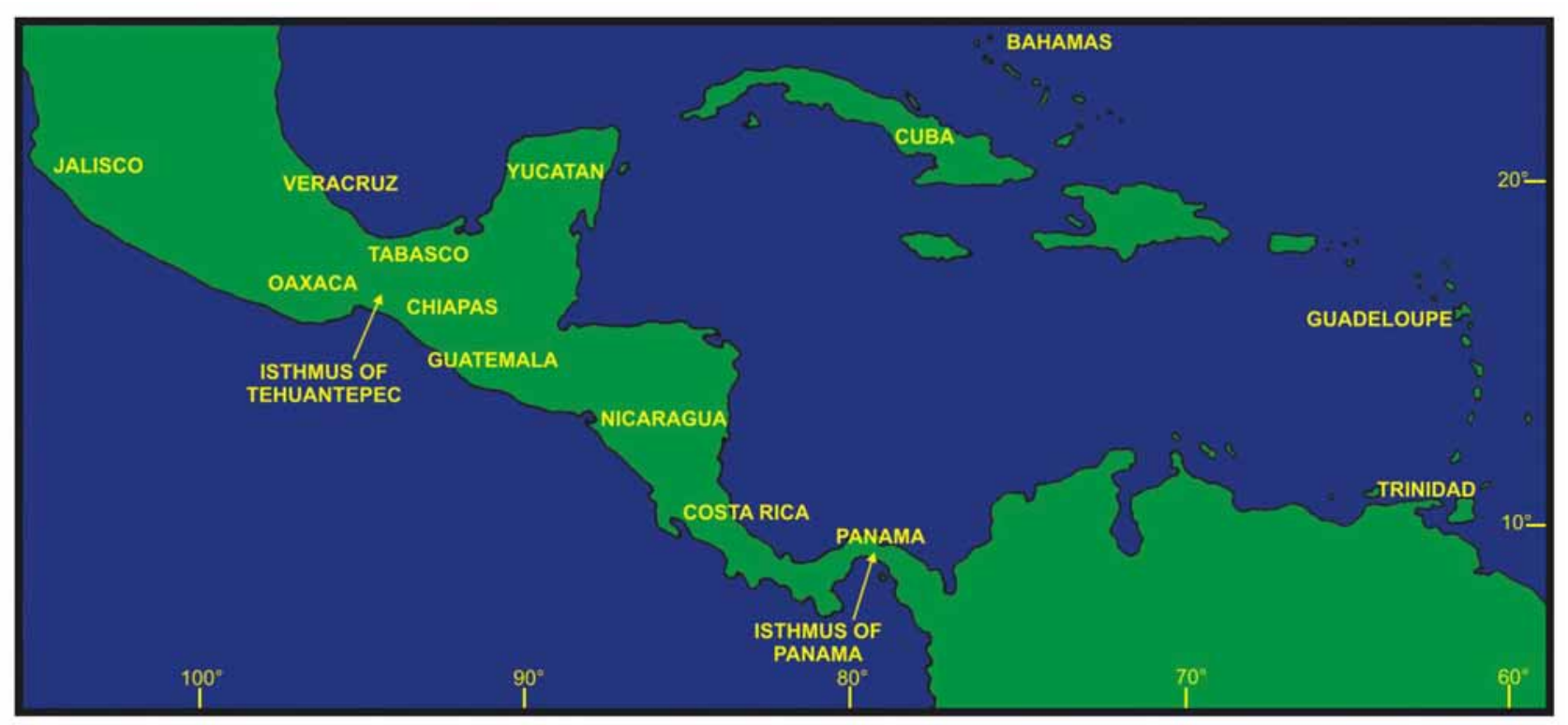

FIGURE 1. Map of Central America and neighbouring areas.

\section{Study area}

Central America (Fig. 1) constitutes the northern most portion of the Neotropics in general terms (MaldonadoKoerdell 1964; Stuart 1966). Bordered by the Pacific Ocean to the south, and the Caribbean Sea and Gulf of Mexico to the northeast, it extends approximately $3000 \mathrm{~km}$ from the Isthmus of Panama northwest to the Isthmus of Tehuantepec. The widest portion $(\sim 1000 \mathrm{~km})$ is in northern Honduras and central Guatemala, and the narrowest is the Panama Canal Zone $(60 \mathrm{~km})$. Overall, it covers $10^{6} \mathrm{~km}^{2}$, most of which is mountainous: $25 \%$ 
is below $600 \mathrm{~m}$ asl (Stuart 1966). Its intrincate pattern of altitude and relief has produced a diverse ecological mosaic in which climate and ecosystem characteristic change abruptly over short distances (Hall \& Pérez-Brignoli 2003). Its northern extent, delimited by the nature of its icthyofauna, is the Río Papaloapan river, Veracruz, Mexico, on the Atlantic coast (Gulf of Mexico) and the Río Tehuantepec river, Oaxaca, Mexico, on the Pacific coast. The Panama region is a transitional zone between the South and Central American fauna (Miller 1966; Bãnãrescu 1995).

The region lacked primary freshwater fish until well into the Tertiary. During the Cenozoic, Central America existed as a peninsula, contiguous with what is today Mexico, and separated from South America by a marine boundary that covered what is currently Nicaragua, Costa Rica and Panama. Central America remained isolated from South America until the Pleistocene. The land bridge between the two continents emerged in the Paleocene and then again in the Pliocene, allowing the dispersion of terrestrial biota (Savage 1966; Rosen 1976, 1985; Bussing 1985; Smith 1985; Donnelly 1989; Coates \& Obando 1996; Bermingham \& Martin 1998). The freshwater systems of Central America, however, did not develop until the Pliocene, about 3 million years ago (Briggs 1995). Because of this physiography and geologic history, Central America has few freshwater fish species, despite being a tropical region (Nelson 2006).

During the Neogene, an ichthyofauna developed in the Central American area that was characterized by its extreme poverty in primary species, strictly freshwater, but dominated by secondary species, that is, fish that generally live in freshwater but can tolerate high salinities and are able to cross lower saline barriers (sensu Myers 1938, but see Rosen 1974 and Sparks \& Smith 2005). The poecilids and cichlids (altogether 139 species), plus a diverse number of species derived from marine species (peripheral fish), are the most numerous fish component found in freshwater habitats in this geographic area (Miller 1966; Stuart 1966; Bussing 1985; Bãnãrescu 1995). The fresh- and brackish-water environments of Central America harbor 456 fish species, 104 primary freshwater fish, 165 secondary fish species and 187 peripheral (Miller 1966; Myers 1966). However recently Bussing (1998) states there are 350 freshwater fish species in Central America.

\section{Materials and methods}

A taxonomic species list of helminth parasites of freshwater fish from Central America was compiled. For the purposes of the present study, only adult helminth forms were included. The list is fundamentally based on current records for the distribution of the helminth parasites of freshwater fish for the part of Mexico corresponding to Central America (Salgado-Maldonado 2006, and references herein) and the remaining Central America counries (Price 1938; Caballero \& Brenes 1957; Brenes 1961; Price \& Bussing 1967, 1968; Kritsky \& Fritts 1970; Kritsky \& Leiby 1972; Watson 1976; de Chambrier \& de Vaucher 1984; Lamothe-Argumedo \& Ponciano-Rodríguez 1986a, b; Scholz et al. 1995a, b, 2004; Moravec 1998; Mendoza-Franco et al. 2000, 2003a, 2004, 2007; Aguirre-Macedo et al. 2001a, b; Aguirre-Macedo \& Scholz 2005; Vidal-Martínez et al. 2001b; Choudhury et al. 2002; González-Solís \& Moravec, 2004). The fauna of the Bahamas and the West Indies is closely related to that of the continent, although that of the Lesser Antilles is primarily South American (Bãnãrescu 1995). For this reason helminths that have been recorded in freshwater fish in the Bahamas, Bermuda, Cuba, Guadeloupe, and Trinidad, in addition to Central America, have been indicated in the list (Vigueras 1936; Mizelle \& Kritsky 1969; Hanek et al. 1974; Kristsky \& Thatcher 1974; Molnar et al. 1974; Petter 1977; Petter et al. 1977; Vinjoy et al. 1985; Moravec \& Coy-Otero 1987; Harris \& Lyles 1992; Moravec \& Salgado-Maldonado 2002; Mendoza-Franco et al. 2006). It is quite possible that the monogenean species that have been reported only on islands to date, but whose host families are also distributed on the continent, may be present in Central America: Curvianchoratus hexacleidus Hanek, Molnar \& Fernando, 1974; Gussevia alii (Molnar, Hanek \& Fernando, 1974); G. cichlasomatis (Molnar, Hanek \& Fernando, 1974); G. dobosi (Molnar, Hanek \& Fernando, 1974); Salsuginus bahamensis (Hanek \& Fernando, 1972); S. bermudae 
Rand \& Wiles, 1987, S. cubensis Mendoza-Franco, Vidal-Martínez, Cruz-Quintana \& Prats-León, 2006, Trinidactylus cichlasomatis Hanek, Molnar \& Fernando, 1974 (see Kritsky et al. 1986); Trinigyrus hypostomatis Hanek, Molnar \& Fernando, 1974; Unilatus unilatus Mizelle \& Kritsky, 1967; "Urocleidoides" corydori Molnar, Hanek \& Fernando, 1974; Urocleidoides curimatae Molnar, Hanek \& Fernando, 1974, "Urocleidoides" kabatai Molnar, Hanek \& Fernando, 1974; "Urocleidoides" margolisi Molnar, Hanek \& Fernando, 1974; "Urocleidoides" trinidadensis Molnar, Hanek \& Fernando, 1974; Gyrodactylus bullatarudis Turnbull, 1956 (see Harris, 1986), Gyrodactylus turnbulli Harris, 1986; the same holds for the nematodes Procamallanus (Spirocamallanus) desettae (Petter, Golvan \& Tcheprakoff, 1977); and Rhabdochona cubensis Moravec \& Coy-Otero, 1987 (see Mizelle \& Kritsky 1969; Hanek \& Fernando 1972; Hanek et al. 1974; Molnar et al. 1974; Petter et al. 1977; Murith \& Beverley-Burton 1985; Moravec \& Coy-Otero 1987; Rand \& Wiles 1987; Harris 1986; Kristsky et al. 1986, 2000; Kohn et al. 2006; Mendoza-Franco et al. 2006). However, inferred records of Central American species were not incorporated in the present analysis.

The criteria for include the species in the list was conservative, and it was not assumed that a species' distribution is limited to Central America unless sufficient data supports this supposition. Taxa recorded in southeast Mexico, but whose position could be modified later with complete taxonomic treatments, were excluded from the list, even though some of these species could be Central American species (e.g. Amphimerus sp., Amphoteromorphus sp., Ancyrocephalinae gen. sp., Dactylogyrus sp., Diplectanidae gen. sp. Gyrodactylus sp., Microcotylidae gen. sp., Proteocephalus sp., among others). Also, three species recorded in freshwater fish, but distributed exclusively in coastal lagoons and brackish environments were not included: Caballerorhynchus lamothei Salgado-Maldonado, 1977; Psudoleptorhynchoides lamothei Salgado-Maldonado, 1977 (Acanthocephala); Vasorhabdochona cablei Martin \& Zam, 1967 (Nematoda) (see Salgado-Maldonado 2006). Also excluded was Johnstonmawsonia sp. from eels from Guadeloupe. The same was true for the helminth species of the tarpon Megalops atlanticus Valenciennes and of the bull shark Carcharinus leucas (Müller \& Henle) because of their vagility and the primarily marine distribution of their hosts (see Watson \& Thorson 1976; Mendoza-Franco et al. 2004). Helminth species recorded only in Atherinopsidae, Goodeidae and Cyprinidae were also not included due to their host families' predominantly Nearctic distribution in the Mexican Higland Plateau (north of $19^{\circ}$ ). Two helminth species recorded from the state of Jalisco, Mexico (north of $19^{\circ}$ ) were not included, Proteocephalus chamelensis Pérez, Brooks \& Berman, 1995 and Procamallanus (Spirocamallanus) jaliscensis Moravec, Salgado-Maldonado \& Caspeta-Mandujano, 2000. Finnally, larval forms and introduced helminth species were excluded. Total richness for the helminth species recorded to date in Central America can be determined by consulting Salgado-Maldonado (2006).

\section{Results}

The list presented here contains 111 adult helminth species reported for Central America to date (Tables 1 and 2). Some helminths have been recorded in fish from a number of families, but their frequency and abundance make it possible to link them unequivocally with a specific family. For example, the trematode Genarchella isabellae (Lamothe-Argumedo, 1977) has been reported in fish from five fish families, including its typical host Rhamdia guatemalensis (Günther) (Heptapteridae) (Lamothe-Argumedo 1977). Current data (SalgadoMaldonado 2006), however, allow it to be clearly associated with the cichlids: it has been recorded in 24 fish species, 19 of which are cichlids. Another example is Neoechinorhynchus golvani Salgado-Maldonado, 1977 reported in 31 fish species from 6 families, but 23 of these species are cichlids.

Data for fish species from 17 families are included in the list, with Cichlidae being the family with the highest number of adult helminth species in Central America ( $S=35$ helminth species). If grouped with the Characidae $(\mathrm{S}=17)$ and Heptapteridae $(\mathrm{S}=11)$, they account for $57 \%$ of the helminth species currently known in the area. A number of helminth species have also been reported in fish from the Poeciliidae $(\mathrm{S}=10)$ and Eleotridae $(\mathrm{S}=5)$. 
Of the 111 adult helminth species of freshwater fish currently known in Central America, 102 (92\%) are endemic to the area, 32 of which can be derived from South American genera. Eight of these species have been reported in South America (Table 2). The monogenean Salsuginus seculus (Mizelle \& Arcadi, 1945) and the nematode Rhabdochona kidderi Pearse, 1936 are the only species already reported from North America (see Hanek \& Fernando, 1972; Moravec, 1998; Moravec et al. 1999; Moravec \& Huffman, 2001). Nematodes were the most abundant group, followed by monogeneans and trematodes (Table 1).

TABLE 1. Taxonomical diversity of helminth parasites of freshwater fishes from Central America.

\begin{tabular}{llll}
\hline & Families & $\begin{array}{l}\text { Genera } \\
\text { Total/Endemics }\end{array}$ & $\begin{array}{l}\text { Species } \\
\text { Total/Endemics }\end{array}$ \\
\hline Trematoda & 9 & $22 / 9$ & $29 / 25$ \\
Monogenea & 2 & $14 / 2$ & $30 / 28$ \\
Cestoda & 3 & $5 / 0$ & $6 / 5$ \\
Acanthocephala & 2 & $2 / 0$ & $2 / 2$ \\
Nematoda & 12 & $21 / 5$ & $44 / 42$ \\
Totals & 28 & $64 / 16$ & $111 / 102$ \\
\hline
\end{tabular}

Of the 65 genera recorded in the area 43 are single species. Those with more than one species are Sciadicleithrum (8 species); Rhabdochona (6); Procamallanus (6); “Urocleidoides" (5); Spinitectus (4); Cucullanus, Genarchella, Gyrodactylus, Saccocoelioides, and Paracapillaria (all with 3); and Campechetrema, Neochasmus, Phyllodistomum, Aphanoblastella, Gussevia, Salsuginus, Proteocephalus, Goezia, Hysterothylacium, Atractis, Orientatractis, and Pseudocapillaria (all with 2 species).

Sixteen of the genera (24\%) are endemic to Central America (nine trematodes, two monogeneans and five nematodes), and 23 (35\%), 11 trematodes, 9 monogeneans, 2 cestodes and one genus of nematode, are South American lineages (Table 2). The genera Salsuginus (Monogenea) and Cystoopsis (Nematoda) are of Nearctic origin (Moravec 1998; Mendoza-Franco \& Vidal-Martínez 2001; Mendoza-Franco et al. 2006). Twenty three genera (15 of nematodes) are broadly distributed. Given that the Ictaluridae and Lepisosteidae are of Nearctic ancestry (Miller 1966; Myers 1966; Bãnãrescu 1995; Miller et al. 2005), the five helminth species recorded in fish from these families were treated as being of Nearctic origin even though their distribution is restricted to Central America.

None of the helminth families recorded to date is exclusive to Central America (Table 2). Those families with the highest number of species are the Dactylogyridae $(S=26)$, Cryptogonimidae $(S=8)$, Capillariidae ( $S$ $=8$ ) and Rhabdochonidae ( $\mathrm{S}=7)$. Fiveteen of the remaining families are represented by two to six species, and ten families by only 1 species. The only suprageneric taxon endemic to Central America to date is the subfamily Neophilometrinae (Nematoda: Philometridae), proposed to include a single genus and species (Moravec et al. 2002).

A total of 11 nematode, two trematodes, and two monogeneans species are distributed exclusively on the Pacific slope of Central America, meaning they have not been collected on the Gulf of Mexico or Caribbean slopes; all of these are endemic to Central America (Table 2). 
TABLE 2. Helminth parasites of freshwater fishes of Central America. Families and genera of helminths listed alphabetically. Geographical distribution in Central America is referred only to the country where each parasite has been recorded. Precise collection locations can be found in the references given for each record from Central American countries, and for records from Mexico from Salgado-Maldonado (2006). ' Genus and species endemic to Central America; ${ }^{2}$ Species recorded from South America; ${ }^{3}$ South American genus, Central American species; ${ }^{4}$ Wide distributed genus, Central American species; ${ }^{5}$ North American genus, Central American species; ${ }^{\mathrm{P}}$ species recorded only from Central American Pacific versant.

TREMATODA

Acanthostomidae Poche, 1926

${ }^{1}$ Atrophecaecum (?) astorquii (Watson, 1976) Lamothe-Argumedo \& Ponciano-Rodríguez, 1986

Site: adults from intestine; metacercariae from fins, occasionally gills, scales, eyes and swimbladder.

Hosts and records: Heptapteridae: Rhamdia nicaraguensis (Günther), Nicaragua (Watson 1976); only metacercariae have been recorded in Mexico from Characidae: Astyanax fasciatus (Cuvier); Cichlidae: Cichlasoma salvini (Günther), C. urophthalmus (Günther), Herichthys pearsei Hubbs, Parachromis managuensis (Günther), Petenia splendida Günther, Rocio octofasciata (Regan), Thorichthys aureus (Günther), T. helleri (Steindachner), T. meeki Brind, T. pasionis (Rivas), Vieja synspila (Hubbs); Clupeidae: Dorosoma sp.; Poeciliidae: Gambusia yucatana Regan, Poecilia latipunctata Meek, P. petenensis Günther, P. velifera (Regan) from Yucatán Peninsula and Tabasco, Mexico.

${ }^{2}$ Pseudoacanthostomum panamense Caballero, Bravo-Hollis \& Grocott, 1953

Site: Intestine

Hosts and records: Ariidae: Sciades seemanni (Günther), Panamá (Caballero, Bravo-Hollis \& Grocott 1953); Ariopsis felis (Linnaeus), Hexanematichthys assimilis (Günther), H. guatemalensis (Günther), Yucatán Peninsula and Tabasco, Mexico.

${ }^{2}$ Stunkardiella minima (Stunkard, 1938) Lamothe-Argumedo \& Ponciano-Rodríguez, 1985

Site: Intestine

Hosts and records: Heptapteridae: Rhamdia nicaraguensis, R. managuensis Nicaragua (Watson 1976); R. laticauda (Kner) Costa Rica (Caballero \& Brenes 1957); Rhamdia guatemalensis (Günther), Yucatán Peninsula, Tabasco and Río Papaloapan Basin, Mexico.

Allocreadiidae Looss, 1902

${ }^{3}$ Auriculostoma astyanace Scholz, Aguirre-Macedo \& Choudhury, 2004

Site: Intestine

Hosts and records: Characidae: Astyanax fasciatus Nicaragua (Scholz et al. 2004)

${ }^{3}$ Creptotrema agonostomi Salgado-Maldonado, Cabañas-Carranza \& Caspeta-Mandujano, 1998

Site: Intestine

Hosts and records: Mugilidae: Agonostomus monticola (Bancroft) Jalisco, and Río Papaloapan Basin, Mexico; Ictaluridae: Ictalurus balsanus (Jordan \& Snyder) Río Balsas Basin, Mexico

Angiodictyidae Looss, 1902

${ }^{1}$ Cichlasotrema ujati Pineda-López \& Andrade-Salas, 1989

Site: Intestine

Hosts and records: Cichlidae: Cichlasoma geddesi (Regan), C. rectangulare, Herichthys pearsei, Petenia splendida Günther, Thorichthys helleri, Vieja bifasciata (Steindachner), V. fenestrata (Günther), V. intermedia (Günther), V. synspila, Tabasco, Chiapas and Yucatán Peninsula, Mexico

Apocreadiidae (Skrajabin, 1942) 
Site: Intestine

Hosts and records: Cichlidae: Cichlasoma sp. Costa Rica (Bravo-Hollis \& Arroyo 1962); Amphilophus rostratus (Gill), A. citrinellum (Günther), A. labiatus (Günther), Cryptoheros spilurum (Günther), Hypsophrys nicaraguensis (Günther), Parachromis managuensis (Günther), Nicaragua (Watson 1976); Cichlasoma tetracantha Cuba (Vinjoy et al. 1985); Vieja maculicauda (Regan), Nicaragua (Aguirre-Macedo et al. 2001b); A. robertsoni (Regan), C. geddesi (Regan), C. mayorum Hubbs, C. rectangulare, C. salvini (Günther), C. urophthalmus, Herichthys cyanoguttatus Baird \& Girard, H. labridens (Pellegrin), H. minckleyi (Kornfield \& Taylor), H. pearsei Hubbs, Oreochromis sp., Parachromis friedrichsthalii (Heckel), P. managuensis, P. motaguensis (Günther), P. splendida, Rocio octofasciata, Theraps lentiginosus (Steindachner), Thorichthys aureus (Günther), T. ellioti Meek, T. helleri, T. meeki, T. pasionis, Vieja argentea (Allgayer), V. bifasciata, V. fenestrata (Günther), V. hartwegi (Taylor \& Miller), V. synspila; Clupeidae: Dorosoma petenense (Günther) from Chiapas, Tabasco, Yucatán Peninsula, Río Papaloapan Basin, Río Pánuco Basin, and Coahuila, Mexico.

Callodistomidae (Odhner, 1910)

\section{${ }^{2}$ Prosthenhystera obesa (Diesing, 1850)}

Site: Gall bladder

Hosts and records: Characidae: Astyanax fasciatus Nicaragua (Aguirre-Macedo et al. 2001b); Ariidae Cathorops melanophus (Günther), Potamarius nelsoni (Evermann \& Goldsborough); Centropomidae: Centropomus undecimalis (Bloch), C. parallelus Poey, Characidae: Astyanax fasciatus , Brycon guatemalensis Regan, Cichlidae: Petenia splendida, Thorychthys helleri, Ictaluridae: Ictalurus furcatus (Valenciennes) from Tabasco, Río Papaloapan Basin, and Yucatán Peninsula, México.

Cryptogonimidae Ward, 1917

${ }^{1}$ Campechetrema herrerai Lamothe-Argumedo, Salgado-Maldonado \& Pineda-López, 1997

Site: Intestine

Hosts and records: Cichlidae: Parachromis friedrichsthalii, Petenia splendida, Rocio octofasciata, Chiapas and Yucatán Peninsula, Mexico.

\section{${ }^{1}$ Campechetrema sp.*}

Site: Intestine

Host and record: Ictaluridae: Ictalurus punctatus (Rafinesque), Veracruz, Mexico.

${ }^{4}$ Neochasmus ackerti Watson, 1976

Site: Intestine

Host and record: Pomadasyidae: Pomadasys crocro (Cuvier), Nicaragua (Watson 1976)

${ }^{4}$ Neochasmus olmecus Lamothe-Argumedo, Pineda-López \& Andrade-Salas, 1989

Site: Intestine

Hosts and records: Centropomidae: Centropomus parallelus, C. undecimalis, Eleotridae: Gobiomorus dormitor Lacepède, Tabasco, Mexico

\section{${ }^{1}$ Oligogonotylus manteri Watson, 1976}

Site: Intestine

Hosts and records: Cichlidae: Amphilophus labiatus (Günther), A. citrinellus (Günther), A. rostratus (Gill), Hypsophrys nicaraguensis (Günther), Parachromis managuensis (Günther), Vieja maculicauda (Regan), Nicaragua (Watson 1976; Aguirre-Macedo et al. 2001b); Cichlidae: Cichlasoma geddesi, C. urophthalmus, Parachromis friedrichsthalii, Petenia splendida, Rocio octofasciata, Thorichthys aureus (Günther), T. helleri, T. meeki, T. pasionis, Vieja synspila, Characidae: Astyanax fasciatus, Clupeidae: Dorosoma petenense (Günther), Megalopidae: Megalops atlanticus Valenciennes, Chiapas, Tabasco, Yucatán Peninsula and Río Papaloapan Basin, Mexico. 
${ }^{1}$ Olmeca laurae Lamothe-Argumedo \& Pineda-López, 1990

Site: Intestine

Hosts and records: Centropomidae: Centropomus parallelus, Characidae: Astyanax fasciatus, Cichlidae: Thorichthys helleri, T. meeki, Tabasco, Mexico.

${ }^{1}$ Pseudocaecincola batallae Lamothe-Argumedo, Salgado-Maldonado \& Pineda-López, 1991

Site: Intestine

Host and record: Cichlidae: Petenia splendida Tabasco and Yucatán Peninsula, Mexico.

${ }^{1}$ Tabascotrema verai Lamothe-Argumedo \& Pineda-López, 1990

Site: Intestine

Hosts and records: Cichlidae: Cichlasoma urophthalmus, Petenia splendid, Thorichthys pasionis, Vieja bifasciata, Tabasco and Yucatán Peninsula, Mexico

Derogenidae Lühe, 1910

${ }^{3}$ Genarchella astyanactis (Watson, 1976) Scholz, Vargas-Vazquez \& Salgado-Maldonado, 1995

Site: Stomach

Host and records: Characidae: Astyanax fasciatus Nicaragua (Watson 1976), from the same host species from Tamaulipas, Río Pánuco Basin and Yucatán Peninsula, Mexico.

${ }^{3}$ Genarchella isabellae (Lamothe-Argumedo, 1977) Kohn, Fernandes, Gibson \& Fróes, 1990

Site: Stomach

Hosts and records: Cichlidae: Cichlasoma geddesi, C. urophthalmus, Herichthys cyanoguttatus, H. minckleyi, H. pearsei, Parachromis friedrichsthalii, P. managuensis, Petenia splendida, Rocio octofasciata, Theraps coeruleus Stawikowski \& Werner, T. lentiginosus, Thorichthys aureus, T. helleri, T. meeki, Vieja argentea, V. bifasciata, V. fenestrata, V. intermedia, V. synspila, Eleotridae: Dormitator maculatus (Bloch), Gobiomorus dormitory Lacépède, Heptapteridae: Rhamdia guatemalensis, Poeciliidae: Gambusia yucatana Regan, Synbranchidae: Ophisternon aenigmaticus Rosen \& Greenwood, Chiapas, Tabasco, Río Papaloapan Basin, and Coahuila, Mexico.

${ }^{3}$ Genarchella tropica (Manter, 1936) Scholz, Vargas-Vazquez \& Salgado-Maldonado, 1995

Site: Stomach

Hosts and records: Heptapteridae: Rhamdia managuensis (Günther), Nicaragua (Watson 1976); Heptapteridae: Rhamdia guatemalensis, Ictaluridae: Ictalurus furcatus, Poeciliidae Gambusia yucatana, Poecilia petenensis (Günther), Veracruz, Tabasco and Yucatán Peninsula, Mexico.

Gorgoderidae Looss, 1901

\section{${ }^{3 ?}$ Dendrorchis sp.*}

Site: Intestine, urinary bladder

Hosts and records: Clupeidae Dorosoma petenese, Goodeidae Ilyodon furcidens (Jordan \& Gilbert), Eleotridae: Eleotris sp., Gobiomorus dormitor Río Papaloapan Basin and Jalisco, Mexico.

${ }^{4}$ Phyllodistomum centropomi Mendoza-Garfías \& Pérez, 2005

Site: Intestine

Host and record: Centropomidae: Centropomus parallelus Río Papaloapan Basin, Mexico.

${ }^{4}$ Phyllodistomum sp.*

Site: Gall bladder

Hosts and records: Centropomidae: Centropomus parallelus, Cichlidae: Cichlasoma geddesi, C. urophthalmus, Parachromis managuensis, Vieja synspila, Eleotridae: Guavina guavina (Valenciennes), Poeciliidae: Heterandria bimacu- 
lata (Heckel) Mexico

Haploporidae Nicoll, 1914

${ }^{3}$ Culuwiya cichlidorum Aguirre-Macedo \& Scholz, 2005

Site: Intestine

Hosts and records: Cichlidae: Vieja maculicauda (Regan), Tomocichla tuba (Meek) Nicaragua; Cichlasoma istlanum (Jordan \& Snyder), C. urophthalmus, Herichthys minckleyi, Oreochromis aureus (Steindachner), O. niloticus, Parachromis managuensis, Petenia splendida, Theraps coeruleus Stawikowski \& Werner, Tilapia sp., Vieja bifasciata, V. synspila, Coahuila, Colima, Jalisco, Tabasco, Chiapas and Yucatán Peninsula, Mexico (Aguirre-Macedo \& Scholz 2005).

${ }^{3}$ Saccocoelioides chauhani Lamothe-Argumedo, 1974

Site: Intestine

Host and record: Characidae: Astyanax fasciatus Río Papaloapan Basin, Mexico

${ }^{3 ?}$ Saccocoelioides cf. sogandaresi Lumsden, 1963

Site: Intestine

Hosts and records: Poeciliidae: Poecilia velifera (Regan) Nicaragua (Aguirre-Macedo et al. 2001b); Poeciliidae: Heterandria bimaculata, Poecilia latipunctata Meek, P. mexicana Steindachner, P. petenensis Günther, P. sphenops Valenciennes, P. velifera, Poeciliopsis catemaco Miller, P. gracilis (Heckel), Xiphophorus hellerii Heckel, Characidae: Astyanax aeneus, Eleotridae: Dormitator maculatus, Gobiomorus dormitor, Gobiidae: Sicydium multipunctatum Regan, Goodeidae: Allodontichthys zonistius (Hubbs), Ilyodon furcidens (Jordan \& Gilbert), I. whitei (Meek), Mugilidae: Agonostomum monticola from Jalisco, Veracruz, Tabasco, Yucatán Peninsula and the basins of the Rivers Papaloapan, Pánuco and Balsas, Mexico.

\section{${ }^{3, \mathrm{P}}$ Saccocoelioides sp.*}

Site: Intestine

Host and record: Eleotridae: Dormitator latifrons Guerrero, Mexico

Macroderoididae McMullen, 1937

\section{${ }^{2}$ Magnivitellinum simplex Kloss, 1966}

Site: Intestine

Hosts and records: Characidae: Astyanax fasciatus Nicaragua (Aguirre-Macedo et al. 2001b), Characidae: A. aeneus, A. mexicanus (De Filippi), Ariidae: Ariopsis felis (Linnaeus), Goodeidae: Ilyodon furcidens Nuevo León, Jalisco, Río Pánuco Basin, Río Papaloapan Basin and Yucatán Peninsula, Mexico.

${ }^{1}$ Perezitrema bychowskyi (Caballero y Caballero, 1975) Brooks, 1980

Site: Intestine

Hosts and records: Lepisosteidae: Atractosteus tropicus Gill, Nicaragua (Watson, 1976), recorded also from the same host species from Tabasco, Mexico. [The conspecificity of this species with Perezitrema viguerasi Baruš \& Moravec recovered from A. tristoechus from Cuba can not be excluded, see Moravec \& Salgado-Maldonado 2002]

${ }^{1 \mathrm{P}}$ Wallinia chavarriae Choudhury, Hartvigsen \& Brooks, 2002

Site: Intestine

Hosts and records: Characidae: Astyanax aeneus, Bryconamericus scleroparius (Regan), Costa Rica (Choudhury, Hartvigsen \& Brooks 2002)

\section{MONOGENEA}

Dactylogyridae Bychowsky, 1933 
Site: Gills

Hosts and records: Heptapteridae: Rhamdia laticauda (Kner), Costa Rica, (Price 1938), R. quelen (Quoy \& Gaimard) Trinidad (Molnar et al. 1974) (see Kritsky et al. 2000), R. nicaraguensis (Günther) Nicaragua (Mendoza-Franco et al. 2003a), R. guatemalensis Tabasco, Yucatán Peninsula and Río Papaloapan Basin, Mexico.

${ }^{3}$ Aphanoblastella chagresii Mendoza-Franco, Aguirre-Macedo \& Vidal-Martínez, 2007

Site: Gills

Host and record: Heptapteridae: Pimelodella chagresi (Steindachner), Panama (Mendoza-Franco et al. 2007)

${ }^{2}$ Aphanoblastella travassosi (Price, 1938) Kritsky, Mendoza-Franco \& Scholz, 2000

Site: Gills

Hosts and records: Heptapteridae: Rhamdia laticauda, Costa Rica, (Price 1938), R. quelen Trinidad (Molnar et al. 1974), R. quelen Panama (Kritsky et al. 2000; Mendoza-Franco et al. 2007), R. nicaraguensis Nicaragua (Mendoza-Franco et al. 2003a), R. guatemalensis Tabasco, Yucatán Peninsula and Río Papaloapan Basin, Mexico.

${ }^{4}$ Dactylogyrus mizellei Price, 1967

Site: Gills

Hosts and records: Poeciliidae: Xiphophorus helleri Guatemala (Price 1967; this species is a junior synonym of D. intermedius Kritsky, personal communication)

${ }^{3}$ Diaphorocleidus petrosusi Mendoza-Franco, Aguirre-Macedo \& Vidal-Martínez, 2007

Site: Gills

Hosts and records: Characidae: Brycon petrosus Meek \& Hildebrand, Panama (Mendoza-Franco et al. 2007)

${ }^{1}$ Guavinella tropica Mendoza-Franco, Scholz \& Cabañas-Carranza, 2003

Site: Gills

Hosts and records: Eleotridae Gobiomorus dormitor Jalisco, Tabasco and Río Papaloapan Basin, Mexico.

${ }^{3}$ Gussevia asota Kritsky, Thatcher \& Boeger, 1989

Site: Gills

Hosts and records: Cichlidae: Astronotus ocellatus (Agassiz), Panama (Mendoza-Franco et al. 2007)

${ }^{3}$ Gussevia heterotilapiae Vidal-Martínez, Scholz \& Aguirre-Macedo, 2001

Site: Gills

Hosts and records: Cichlidae: Heterotilapia multispinosa (Günther) Nicaragua (Vidal-Martínez et al. 2001b)

3?P Jainus hexops Kritsky \& Leiby, 1972

Site: Gills

Hosts and records: Characidae: Astyanax fasciatus Costa Rica (Kritsky \& Leiby 1972)

${ }^{2}$ Palombitrema heteroancistrium Price \& Busing, 1968

Site: Gills

Hosts and records: Characidae: Astyanax fasciatus Costa Rica (Price \& Busing 1968), collected from the same host in Nicaragua (Mendoza-Franco et al. 2003a); Characidae: Astyanax aeneus, Cichlidae: Cichlasoma urophthalmus Tabasco and Yucatán Peninsula, Mexico. 
${ }^{3 ?}$ Pavanelliella scaphiocotylus Kritsky \& Mendoza-Franco, 2003

Site: Nasal cavity

Host and record: Heptapteridae: Rhamdia guatemalensis Yucatán Peninsula, Mexico

${ }^{5}$ Salsuginus neotropicalis Mendoza-Franco \& Vidal-Martínez, 2001

Site: Gills

Host and records: Poeciliidae: Belonesox belizanus Kner, Nicaragua (Mendoza-Franco et al. 2003a), collected from the same host in Yucatán Peninsula, Mexico.

${ }^{5}$ Salsuginus seculus (Mizelle \& Arcadi, 1945) Fleurish \& Beverley-Burton, 1985

Site: Gills

Hosts and records: Poeciliidae: Gambusia affinis (Baird \& Girard), Bahamas (Hanek \& Fernando 1972), G. yucatana Tabasco, Mexico. This species has been previously recorded from North America (see Hanek \& Fernando 1972).

${ }^{3}$ Sciadicleithrum bicuense Vidal-Martínez, Scholz \& Aguirre-Macedo, 2001

Site: Gills

Hosts and records: Cichlidae: Archocentrus nigrofasciatus Günther, Nicaragua (Vidal-Martínez et al. 2001b)

Cichlidae (1); Nicaragua

${ }^{3}$ Sciadicleithrum bravohollisae Kritsky, Vidal-Martínez \& Rodríguez-Canul, 1994

Site: Gills

Hosts and records: Cichlidae: Vieja maculicauda (Regan) Nicaragua (Mendoza-Franco et al. 2003a), Amphilophus citrinellus, Cichlasoma geddesi, C. salvini, C. urophthalmus, Herichthys labridens, H. pearsei, Oreochromis aureus, Parachromis managuensis, Petenia splendida, Rocio octofasciata, Theraps lentiginosus, Thorichthys helleri, Vieja fenestrata, V. synspila from Río Pánuco Basin, Tabasco, Chiapas, Río Papaloapan Basin and Yucatán Peninsula, Mexico.

${ }^{3}$ Sciadicleithrum maculicaudae Vidal-Martínez, Scholz \& Aguirre-Macedo, 2001

Site: Gills

Hosts and records: Cichlidae: Cichlasoma maculicauda (Regan) Nicaragua (Vidal-Martínez et al. 2001b)

${ }^{3}$ Sciadicleithrum meekii Mendoza-Franco, Scholz \& Vidal-Martínez, 1997

Site: Gills

Hosts and records: Cichlidae: Archocentrus nigrofasciatus Günther, Nicaragua (Vidal-Martínez et al. 2001b; MendozaFranco et al. 2003a), Parachromis managuensis, Thorichthys helleri, T. meeki, T. callolepis Tabasco, Chiapas and Yucatán Peninsula, Mexico.

${ }^{3}$ Sciadicleithrum mexicanum Kritsky, Vidal-Martínez, \& Rodríguez-Canul, 1994

Site: Gills

Hosts and records: Cichlidae: Cichlasoma trimaculatum (Günther) Guatemala (Mendoza-Franco et al. 2000) $C$. urophthalmus, Parachromis dovii (Günther), P. managuensis, Tomocichla tuba (Meek), Vieja maculicauda Nicaragua (Vidal-Martínez et al. 2001b, Mendoza-Franco et al. 2003a), C. urophthalmus, Parachromis friedrichsthalii, Petenia splendid, Rocio octofasciata, Thorynchthys aureus, Vieja sysnpila Chiapas, Tabasco and Yucatán Peninsula, Mexico.

${ }^{3}$ Sciadicleithrum nicaraguense Vidal-Martínez, Scholz \& Aguirre-Macedo, 2001

Site: Gills

Hosts and records: Cichlidae: Amphilophus alfari (Meek) Nicaragua (Vidal-Martínez et al. 2001b, Mendoza-Franco et 
al. 2003a)

${ }^{3}$ Sciadicleithrum panamensis Mendoza-Franco, Aguirre-Macedo, \& Vidal-Martínez, 2007

Site: Gills

Hosts and records: Cichlidae: Aequidens coeruleopunctatus (Kner) Panama (Mendoza-Fanco et al. 2007)

${ }^{3}$ Sciadicleithrum splendidae Kritsky, Vidal-Martínez, \& Rodríguez-Canul, 1994

Site: Gills

Hosts and records: Cichlidae: Parachromis friedrichsthalii, P. managuensis, Petenia splendida, Vieja synspila Chiapas, Tabasco and Yucatán Peninsula, Mexico.

36Urocleidoides" costaricensis (Price \& Bussing, 1967) Kritsky \& Leiby, 1972

Site: Gills

Hosts and records: Characidae: Astyanax fasciatus Costa Rica (Price \& Bussing 1967; Kritsky \& Leiby 1972), A. fasciatus and Curimatidae: Steindachneria argentea (Gill) Trinidad (Molnar et al. 1974), A. fasciatus Nicaragua (Mendoza-Franco et al. 2003a) from the same host from Río Balsas and Río Papaloapan Basins, Tabasco and Yucatán Peninsula, Mexico.

${ }^{3}$ Urocleidoides flegomai Mendoza-Franco, Aguirre-Macedo, \& Vidal-Martínez, 2007

Site: Gills

Hosts and records: Lebiasinidae: Piabucina panamensis Gill Panama (Mendoza-Franco et al. 2007)

${ }^{3}$ Urocleidoides reticulatus Mizelle \& Price, 1964

Site: Gills

Hosts and records: Poeciliidae: Poecilia reticulata Trinidad (Kritsky et al. 1986), Belonesox belizanus, Poecilia mexicana, P. petenensis Tabasco, Mexico.

${ }^{3}$ Urocleidoides similuncus Mendoza-Franco, Aguirre-Macedo, \& Vidal-Martínez, 2007

Site: Gills

Hosts and records: Poeciliidae: Poecilia gillii (Kner) Panama (Mendoza-Franco et al. 2007)

36Urocleidoides" strombicirrus (Price \& Bussing, 1967) Kritsky \& Thatcher, 1974

Site: Gills

Hosts and records: Characidae: Astyanax fasciatus Costa Rica (Price \& Bussing, 1967), from the same host from Nicaragua (Mendoza-Franco et al. 2003a), A. fasciatus, A. mexicanus Tabasco and Río Pánuco Basin, Mexico.

Gyrodactylidae

${ }^{1}$ Anacanthocotyle anacanthocotyle Kritsky \& Fritts, 1970

Site: Gills

Hosts and records: Characidae: Astyanax fasciatus Costa Rica (Kritsky \& Fritts 1970), A. aeneus, A. fasciatus Río Papaloapan Basin and Yucatán Peninsula, Mexico.

${ }^{4}$ Gyrodactylus bullatarudis Turnbull, 1956

Site: Gills

Hosts and records: Poeciliidae: Poecilia sphenops Costa Rica (Kritsky \& Fritts 1970), P. reticulata Trinidad (Harris \& Lyles 1992) 
${ }^{4, \mathrm{P}}$ Gyrodactylus costaricensis Kristky \& Fritts, 1970

Site: Gills

Hosts and records: Poeciliidae: Poecilia sphenops Costa Rica (Kritsky \& Fritts 1970)

${ }^{4}$ Gyrodactylus neotropicalis Kritsky \& Fritts, 1970

Site: Gills

Host and records: Characidae: Astyanax fasciatus Costa Rica (Kritsky \& Fritts 1970), from the same host species from Yucatán Peninsula, Mexico.

\section{CESTODA}

Bothriocephalidae

${ }^{4}$ Bothriocephalus pearsei Scholz, Vargas-Vázquez \& Moravec, 1996

Site: Intestine

Hosts and records: Cichlidae: Cichlasoma urophthlmus, Heptapteridae: Rhamdia guatemalensis Yucatán Peninsula, Mexico.

Monticelliidae La Rue, 1911

${ }^{2 ?}$ Amphoteromorphus sp.

Site: Intestine

Hosts and records: Centropomidae: Centropomus parallelus, C. undecimalis Tabasco, Mexico.

${ }^{3}$ Monticellia ophisterni Scholz, de Chambrier \& Salgado-Maldonado, 2001

Site: Intestine

Hosts and records: Synbranchidae: Ophisternon aenigmaticum Rosen \& Greenwood Río Papaloapan Basin, Mexico.

Proteocephalidae La Rue, 1911

${ }^{5}$ Megathylacoides lamothei (García-Prieto, 1990)

Site: Intestine

Hosts and records: Ictaluridae: Ictalurus furcatus Veracruz and Río Papaloapan Basin, Mexico; I. balsanus, I. furcatus Tabasco and Michoacán, Mexico (Rosas-Valdez et al. 2004).

${ }^{4}$ Proteocephalus brooksi García-Prieto, Rodríguez \& Pérez, 1996

Site: Intestine

Hosts and records: Heptapteridae: Rhamdia guatemalensis Río Papaloapan Basin and Yucatán Peninsula, Mexico.

${ }^{4}$ Proteocephalus gaspari de Chambrier \& Vaucher, 1984

Site: Intestine

Hosts and records: Lepisosteidae: Atractosteus tropicus Nicaragua (de Chambrier \& Vaucher 1984)

\section{ACANTHOCEPHALA}

Echinorhynchidae Cobbold, 1876

\section{${ }^{4}$ Acanthocephalus sp. 1*}

Site: Intestine

Hosts and records: Cichlidae: Cichlasoma urophthalmus Yucatán Peninsula, Mexico.

Neoechinorhynchidae Ward, 1917 
Site: Intestine

Hosts and records: Cichlidae: Amphilophus alfari Nicaragua (Aguirre-Macedo et al. 2001b), Ariidae: Ariopsis felis (Linnaeus), Cathorops aguadulce (Meek), Belonidae: Strongylura sp., Cichlidae: Amphilophus robertsoni, Archocentrus nigrofasciatus, Cichlasoma beani, C. geddesi, C. istlanum, C. rectangulare, C. salvini, C. urophthalmus, Herichthys cyanoguttatus, H. labridens, H. pearsei, Parachromis friedrichsthalii, P. managuensis, P. motaguensis, Petenia splendida, Rocio octofasciata, Thorichthys ellioti, T. helleri, T. meeki, T. pasionis, Vieja fenestrata, V. synspila, Eleotridae: Dormitator latifrons, D. maculatus, Gobiomorus dormitor, Heptapteridae: Hyporhamphus mexicanus, Ictaluridae: Ictalurus furcatus Guerrero, Veracruz, Tabasco, Río Papaloapan and Río Pánuco Basins, and Yucatán Peninsula, Mexico.

\section{NEMATODA}

Anisakidae Railliet \& Henry, 1912

${ }^{4, \mathrm{P}}$ Goezia nonipapillata Osorio-Sarabia, 1982

Site: Intestine

Hosts and records: Cichlidae: Cichlasoma istlanum (Jordan \& Snyder), Oreochromis aureus, O. mossambicus, Tilapia zilii Río Balsas Basin, Mexico.

\section{${ }^{4 ?}$ Goezia sp.*}

Site: Intestine

Hosts and records: Cichlidae: Vieja hartwegi, Ictaluridae: Ictalurus dugesii, I. balsanus Chiapas and Río Balsas Basin, Mexico.

${ }^{4}$ Hysterothylacium cenotae (Pearse, 1936)

Site: Intestine

Hosts and records: Heptapteridae: Rhamdia guatemlensis Yucatán Peninsula, Mexico.

${ }^{4, \mathrm{P}}$ Hysterothylacium perezi Gopar-Merino, Osorio-Sarabia \& García-Prieto, 2005

Site: Intestine

Hosts and records: Ariidae: Sciades guatemalensis (Günther) Guerrero, Mexico.

Atractidae Railliet, 1917

${ }^{4, \mathrm{P}}$ Atractis bravoae (Osorio-Sarabia, 1984) Moravec, 2000

Site: Intestine

Hosts and records: Atherinopsidae: Atherinella balsana (Meek), Cichlidae: Cichlasoma istlanum Río Balsas Basin, Mexico.

${ }^{4}$ Atractis vidali González-Solís \& Moravec, 2002

Site: Intestine

Hosts and records: Cichlidae: Herichthys pearsei, Vieja intermedia Chiapas and Yucatán Peninsula, Mexico.

${ }^{4}$ Orientatractis campechensis González-Solís \& Moravec, 2004

Site: Intestine

Hosts and records: Cichlidae: Herichthys pearsei, Vieja bifasciata Chiapas and Yucatán Peninsula, Mexico.

${ }^{4}$ Orientatractis chiapasensis González-Solís \& Moravec, 2004

Site: Intestine 
Hosts and records: Cichlidae: Tomocichla tuba (Meek) Nicaragua (González-Solís \& Moravec 2004), Vieja intermedia, Chiapas, Mexico.

Camallanidae Railliet \& Henry, 1915

${ }^{4}$ Procamallanus (Spirocamallanus) chetumalensis González-Solís, Moravec \& Vidal-Martínez, 2002

Site: Intestine

Hosts and records: Ariidae: Sciades assimilis (Günther), Yucatán Peninsula, Mexico (González-Solís et al. 2002)

${ }^{4, \mathrm{P}}$ Procamallanus (Spirocamallanus) gobiomori Moravec, Salgado-Maldonado \& Caspeta-Mandujano, 2000

Site: Intestine

Hosts and records: Eleotridae: Dormitator maculatus (Bloch), Eleotris picta Kner, Gobiomorus polylepis Ginsburg, Jalisco, Mexico.

${ }^{4, \mathrm{P} P r o c a m a l l a n u s}$ (Spirocamallanus) jaliscensis Moravec, Salgado-Maldonado \& Caspeta-Mandujano, 2000

Site: Intestine

Host and record:Mugilidae: Agonostomus monticola (Bancroft) Jalisco, Mexico

${ }^{4}$ Procamallanus (Spirocamallanus) mexicanus Moravec, Salgado-Maldonado \& Caspeta-Mandujano, 2000

Site: Intestine

Hosts and records: Cichlidae: Cichlasoma geddesi (Regan), Veracruz, Mexico.

${ }^{4}$ Procamallanus (Spirocamallanus) neocaballeroi (Caballero-Deloya, 1977)

Site: Intestine

Hosts and records: Cichlidae: Vieja maculicauda (Regan), Nicaragua (Aguirre-Macedo et al. 2001b); Characidae: Astyanax fascitus, A. mexicanus, Bramocharax caballeroi, Heptapteridae: Rhamdia guatemalensis, Tabasco, Yucatán Peninsula, and Río Pánuco and Río Papaloapan Basins, Mexico, Nicaragua

${ }^{4}$ Procamallanus (Spirocamallanus) rebecae (Andrade-Salas, Pineda-López \& García- Magaña, 1994)

Site: Intestine

Hosts and records: Cichlidae: Amphilophus alfari (Meek), Heterotilapia multispinosa, Vieja maculicauda, Nicaragua (Aguirre-Macedo et al. 2001b); Cichlidae: Amphilophus citrinellus (Günther), A. robertsoni (Regan), Cichlasoma geddesi, C. rectangulare, C. urophthalmus, Parachromis managuensis, P. motaguensis (Günther), Petenia splendida, Thorichthys callolepis T. helleri (Steindachner), T. meeki Brind, T. pasionis (Rivas), Vieja fenestrata (Günther), V. synspila (Hubbs), Belonidae: Strongylura sp., Characidae: Astyanax fasciatus, Centropomidae: Centropomus parallelus Tabasco, Yucatán Peninsula and Río Papaloapan Basin, Mexico.

Capillariidae Railliet, 1915

${ }^{4}$ Capillaria (Hepatocapillaria) cichlasomae Moravec, Scholz \& Mendoza-Franco, 1995

Site: Intestine

Host and record: Cichlidae: Cichlasoma urophthalmus Yucatán Peninsula, Mexico

${ }^{4 ?}$ Capillostrongyloides sp.

Site: Intestine

Host and record: Characidae: Astyanax fasciatus Yucatán Peninsula, Mexico

${ }^{4}$ Paracapillaria rhamdiae Moravec, González-Solís \& Vargas-Vázquez, 1995

Site: Intestine

Host and record: Heptapteridae: Rhamdia guatemalensis Yucatán Peninsula, Mexico. 
Site: Intestine

Hosts and records: Eleotridae: Dormitator maculatus, Gobiomorus dormitor, Tabasco, Veracruz, Río Papaloapan Basin and Yucatán Peninsula, Mexico.

${ }^{4, \mathrm{P}}$ Paracapillaria teixeirafreitasi pacifica Moravec, Salgado-Maldonado \& Caspeta-Mandujano, 1999

Site: Intestine

Hosts and records: Eleotridae: Dormitator maculatus, Gobiomorus polylepis, Jalisco, Mexico.

${ }^{1, \mathrm{P}}$ Paracapillaroides agonostomi Moravec, Salgado-Maldonado \& Caspeta-Mandujano, 1999

Site: Intestine

Host and record: Mugilidae: Agonostomus monticola, Jalisco, Mexico.

${ }^{4}$ Pseudocapillaria (Ichthyocapillaria) ophisterni Moravec, Salgado-Maldonado \& Jiménez-García, 2000

Site: Intestine

Host and record: Synbranchidae: Ophisternon aenigmaticum Río Papaloapan Basin, Mexico.

${ }^{4}$ Pseudocapillaria yucatanensis Moravec, Scholz \& Vivas-Rodríguez, 1995

Site: Intestine

Host and record: Heptapteridae: Rhamdia guatemalensis, Yucatán Peninsula, Mexico.

Cosmocercidae Railliet, 1916

${ }^{4}$ Raillietnema kritscheri Moravec, Salgado-Maldonado \& Pineda-López, 1993

Site: Intestine

Hosts and records: Cichlidae: Cichlasoma urophthalmus, Herichthys pearsei, Vieja synspila, Chiapas, Tabasco and Yucatán Peninsula, Mexico.

Cucullanidae Cobbold, 1864

${ }^{4}$ Dichelyne mexicanus Caspeta-Mandujano, Moravec \& Salgado-Maldonado, 1999

Site: Intestine

Hosts and records: Cichlidae: Cichlasoma beani (Jordan), Ictaluridae: Ictalurus balsanus, I. furcatus, I. punctatus, Mugilidae: Agonostomus monticola, Tamaulipas, Veracruz, and basins of the rivers Balsas, Paploapan and Santiago, Mexico.

${ }^{4}$ Cucullanus angeli Cabañas-Carranza \& Caspeta-Mandujano, 2007

Site: Intestine

Host and record: Cichlidae: Vieja intermedia Chiapas, Mexico.

${ }^{4}$ Cucullanus (Cucullanus) caballeroi Petter, 1977

Site: Intestine

Hosts and records: Eleotridae: Eleotris pisonis (Gmelin), Gobiomorus maculatus Guadeloupe (Petter 1977), Cichlidae: Cichlasoma sp., Eleotridae: Dormitator maculatus, Gobiomorus dormitor, Heptapteridae: Rhamdia guatemalensis, Río Papaloapan Basin, Mexico.

${ }^{4}$ Cucullanus mexicanus Caspeta-Mandujano, Moravec \& Aguilar-Aguilar, 2000

Site: Intestine

Host and records: Heptapteridae: Rhamdia guatemalensis Río Papaloapan Basin, Mexico. 
Site: Intestine

Host and record: Characidae: Brycon guatemalensis Chiapas, Mexico.

Cystidicolidae Skrjabin, 1946

${ }^{4}$ Spinitectus agonostomi Moravec \& Barus, 1971

Site: Intestine

Host and records: Mugilidae: Agonostomus monticola Cuba and Guadeloupe (see Moravec 1998), and from the same host species from Jalisco and Río Papaloapan Basin, Mexico.

${ }^{4, \mathrm{P}}$ Spinitectus humbertoi Caspeta-Mandujano \& Moravec, 2000

Site: Intestine

Host and record: Profundulidae: Profundulus labialis (Günther), Guerrero, Mexico.

${ }^{4}$ Spinitectus mexicanus Caspeta-Mandujano, Moravec \& Salgado-Maldonado, 2000

Site: Intestine

Host and record: Poeciliidae: Heterandria bimaculata (Heckel), Poecilia mexicana Steindachner, Río Papaloapan Basin, Mexico.

${ }^{4}$ Spinitectus tabascoensis Moravec, García-Magaña \& Salgado-Maldonado, 2002

Site: Intestine

Host and record: Ictaluridae: Ictalurus furcatus Tabasco, Mexico.

Cystoopsidae Maggenti 1981

${ }^{5}$ Cystoopsis atractostei Moravec \& Salgado-Maldonado, 2003

Site: Intestine

Host and records: Lepisosteidae: Atractosteus tropicus Tabasco, Mexico.

Daniconematidae Moravec \& Køie, 1987

${ }^{1}$ Mexiconema cichlasomae Moravec, Vidal \& Salgado-Maldonado, 1992

Site: Intestine

Hosts and records: Cichlidae: Cichlasoma urophthalmus, Herichthys pearsei, Parachromis motaguensis, Thorichthys helleri, T. pasionis, Vieja synspila, Ariidae: Cathorops melanopus (Günther), Poeciliidae: Xiphophorus hellerii Heckel, Río Papaloapan Basin, Tabasco and Yucatán Peninsula, Mexico.

Philometridae Baylis \& Daubney, 1926

${ }^{1}$ Neophilometroides caudatus (Moravec, Scholz \& Vivas-Rodríguez, 1995) Moravec, Salgado-Maldonado \& AguilarAguilar, 2002

Site: Intestine

Host and records: Heptapteridae: Rhamdia guatemalensis Río Papaloapan Basin and Yucatán Peninsula, Mexico.

${ }^{4}$ Philometra ophisterni Moravec, Salgado-Maldonado \& Aguilar-Aguilar, 2002

Site: Intestine

Host and record: Synbranchidae : Ophisternon aenigmaticum Río Papaloapan Basin, Mexico.

Quimperiidae Gendre, 1928

'Gibsonnema ophisterni (Moravec, Salgado-Maldonado \& Aguilar-Aguilar, 2002) Moravec, Salgado-Maldonado \& Aguilar-Aguilar, 2002 
Site: Intestine

Host and record: Synbranchidae: Ophisternon aenigmaticum Río Papaloapan Basin, Mexico.

Rhabdochonidae Travassos, Artigas \& Pereira, 1928

${ }^{1, \mathrm{P}}$ Beaninema nayaritense Caspeta-Mandujano, Moravec \& Salgado-Maldonado, 2001

Site: Intestine

Host and record: Cichlidae: Cichlasoma beani, Río Santiago Basin, Mexico.

${ }^{2}$ Rhabdochona acuminata (Molin, 1860)

Site: Intestine

Host and record: Characidae: Brycon guatemalensis, Chiapas, Mexico.

${ }^{4, \mathrm{P}}$ Rhabdochona guerreroensis Caspeta-Mandujano, Aguilar-Aguilar \& Salgado-Maldonado, 2002

Site: Intestine

Host and record: Gobiidae: Sicydium multipunctatum, Guerrero and Jalisco, Mexico.

${ }^{4}$ Rhabdochona kidderi Pearse, 1936

Site: Intestine

Hosts and records: Cichlidae: Vieja maculicauda, Nicaragua (Aguirre-Macedo 2001b); Bythidae: Typhliasina pearsei (Hubbs), Cichlidae: Amatitlania nigrofasciata (Günther), Cichlasoma istlanum, C. urophthalmus, Herichthys cyanoguttatus, H. labridens, Parachromis managuensis, Thorichthys helleri, Vieja bifasciata, V. fenestrata, V. synspila, Clupeidae: Dorosoma anale Meek, Eleotridae: Gobiomorus dormitor, Heptapteridae: Rhamdia guatemalensis, Ictaluridae: Ictalurus mexicanus (Meek), Poeciliidae: Gambusia yucatana Regan, Poecilia mexicana, basins of the rivers Balsas, Pánuco and Papaloapan, Jalisco, Tabasco and Yucatán Peninsula, Mexico.

${ }^{4}$ Rhabdochona mexicana Caspeta-Mandujano, Moravec \& Salgado-Maldonado, 2000

Site: Intestine

Hosts and records: Characidae: Astyanax aeneus, A. fasciatus, A. mexicanus (De Filippi), basins of the rivers Balsas, Pánuco and Papaloapan, and Jalisco, Mexico.

${ }^{4, \mathrm{P}}$ Rhabdochona salgadoi Caspeta-Mandujano \& Moravec, 2000

Site: Intestine

Host and record: Profundulidae: Profundulus labialis, Guerrero, Mexico.

${ }^{4}$ Rhabdochona xiphophori Caspeta-Mandujano, Moravec \& Salgado-Maldonado, 2001

Site: Intestine

Host and record: Goodeidae: Allotoca catarinae (de Buen), Xenotoca eiseni (Rutter), Poeciliidae: Xiphophorus helleri, Xiphophorus sp. Colima and basins of the rivers Balsas, Pánuco and Santiago, Mexico.

* New species, see Salgado-Maldonado 2006

\section{Discussion}

According to the data the helminth parasite fauna in freshwater fish in Central America is typical to the region. Most of the known species are endemic to the area and can be derived from South American genera. As is the case with mollusks, aquatic insects, crustaceans and freshwater fish (Stehli \& Webb 1985; Bãnãrescu 1995), the number of South American taxa far outnumbers those from North America.

Several authors suggested that the Central American helminth parasite fauna in freshwater fish developed 
allopatrically in the area. They assumed that the ancestral fish stocks that colonized the area brought their ancestral parasites with them and then both hosts and parasites developed in isolation in Central America (Vidal-Martínez \& Kennedy 2000; Vidal-Martínez et al. 2001b; Mendoza-Franco et al. 2007). The isolation of some bodies of water, such as the sinkholes (cenotes) of the Yucatan Peninsula, provided them with peculiar biotic and abiotic conditions that favored speciation processes, including differentiation of endemic forms (Moravec et al. 1995b; Scholz et al. 1996b). Apparently, the Central American helminth fauna developed primarily from radiation of migrants in a competition-free environment, diversifying opportunistically to occupy available niches.

The almost total absence of differentiation of endemic suprageneric taxa, as well as the relatively low number of endemic helminth genera (24\%) suggest this fauna is young. In Central America, the Ichthtyofauna is primarily composed of generalized fish genera which are broadly distributed in South America (Myers 1966; Bãnãrescu 1995). This is also the case with the area's helminth genera, of which a large proportion (35\%) originated from genera that are broadly distributed and dominant in South America, where this fauna is rich and diverse. For example, the genera Gussevia and Sciadicleithrum (Monogenea), and Procamallanus and Spinitectus (Nematoda) are all South American and have the highest numbers of species in this region (Moravec 1998; Kritsky et al. 1994; Mendoza-Franco et al. 2000; Vidal-Martínez et al. 2001b).

There are very few North American freshwater fish in Central America and all are restricted to the extreme north (Myers 1966; Bãnãrescu 1995). Records exist, however, of helminth species in both the Lepisosteidae and Ictaluridae that are found in no other geographic area.

Dispersion and vicariance events that affected their hosts are what determined the current distribution of direct lifecycle parasites (Bentz et al. 2006). It is to be expected, therefore, that the direct lifecycle helminth groups are those that have experienced extensive diversification, resulting in a high number of species. The large number of dactylogyridae monogeneans, particularly Sciadicleithrum, supports this. Monogenean diversity in Central America is most likely higher than reported here, but the study of these parasites in this area is still incipient. The same phenomenon explains how some direct lifecycle nematode, such as the Atractidae, Cosmocercidae and some Capillaridae (Moravec 1998; Anderson 2000), have produced a large number of endemic species in Central America.

Indirect lifecycle helminths have had to confront the challenge of finding appropriate intermediate hosts in Central America. Camallanidae and Rhabdochonidae, the nematode families with the largest number of known species in the region, use broadly distributed and abundant copepods or aquatic insect larvae (Moravec et al. 1995b; Moravec 1998). Specificity in the nematode parasites of fish is much broader in their intermediary hosts than in their definitive hosts (Moravec 1994; Moravec et al. 1995a; Moravec \& Vargas-Vazquez 1996).

Given the above, the current recorded trematode diversity in Central America is surprising. The data of Scholz et al. (1994, 1995a, 1996a) and Ditrich et al. (1996) suggest that a number of trematode lineages used intermediate hosts that were broadly distributed in the area and abundant in some locations. The prosobranchia Pyrgophorus coronatus (Pfeiffer) is thus used as intermediate host by 11 trematode species on the Yucatan Peninsula (Ditrich et al. 1997). The freshwater mollusk fauna here is depauperate, but this snail is a typical component in cenotes and predominates at almost all locations.

The virtual absence of acanthocephalans in Central America (Salgado-Maldonado et al. 1992) cannot be easily explained. Contrary to what Pérez \& Choudhury (2005) erroneously stated the Characidae, Cichlidae and Heptapteridae (= Pimelodidae) as well as other Southamerican fish families do possess typical acanthocephalans in their ancestral area. As for example several species of Echinorhynchus, and Palliolisentis and Neoechinorhynchus buttnerae Golvan, 1956, and Quadrigyrus torquatus Van Cleave, 1920 from Characidae; N. paraguayensis Machado-Filho, 1959, Pandosentis iracundus Van Cleave, 1920 and Q. torquatus from Cichlidae and Deltacanthus scorzai (Díaz-Ungría \& Gracias-Rodrigo, 1957) (= Acanthodelta scorzai) from Pimelodidae (see Thatcher, 2006). Therefore the paucity of acanthocephalans in Mexican freshwaters can not 
be explained as primary. It is most likely that the possible intermediate hosts for acanthocephalans in Central America are in fact highly diverse, but occur in very low abundance populations and are distributed microendemically. They therefore constitute a limiting factor for diversification of the acanthocephalan parasites of freshwater fish. In effect the peracarids and decapods are the most diverse groups of freshwater crustaceans in Mexico and consist mostly of microendemic species. Of the 184 known decapod species from Mexico and Central America, 153 are found in Mexico: 140 of these are endemic; 114 are distributed in a single location; and 22 of these genera are monotypical and endemic. In other words, the region's crustacean species are extremely rare, distributed in very few bodies of water and with populations containing few individuals (Álvarez, F. 2008, Curator, Colección Nacional de Crustáceos, Mexico, Instituto de Biología, Universidad Nacional Autónoma de México). For ancestral acanthocephalan stocks, this would have been the worst possible scenario to find upon arriving in Central America, no matter their origins. Due to the similarity of their lifecycles with acanthocephalan lifecycles, this explanation could also be assumed for cestodes.

A striking feature of this helminth fauna relates to the number of species of parasites associated to the most abundant fish families of Central America, the cichlids and the poeciliids. The Poeciliidae is the only fish family that has given rise to genera and suprageneric taxa endemic to Central America (Myers 1966; Rosen 1975; Bãnãrescu 1995); 56 species of Poeciliids are found in Central America (Myers 1966), however, there are records for only nine helminth species associated to this family. In contrast, only six to nine genera, but more than 100 species of Cichlidae are found in Central America (Miller 1966; Bãnãrescu 1995; Roe et al. 1997; Miller \& Norris 2005), however, cichlids have a record of 33 helminth species.

As shown here, the helminth parasites of freshwater fish from Central America is a fauna that has been almost unable to invade Nearctic regions further north than the Transverse Volcanic Axis (approx. the $19^{\circ}$ parallel) and is not found in South America.

The present data suggest that Central America is a center of diversification (sensu Minckley et al., 2005) for the helminth parasites of freshwater fish. Central America has a typical helminth parasite fauna in freshwater fish with a high proportion of endemisms, that seems evolutionarily very recent. This said, the current state of knowledge of this fauna is incipient and further collections and basic research are needed to better understand it. For example, the differences between genera and species groups with similar distribution patterns (i.e. elements; sensu Bussing 1976) needs more research, as do the distribution patterns of helminths that also adapt to ichthyological provinces (Miller 1966; Bussing 1976). An understanding of the helminth fauna of freshwater fish in Central America will provide an adequate and robust reference framework for future research on the biogeography and ecology of these parasites.

\section{Acknowledgments}

Financial support for this study was provided by the Universidad Nacional Autónoma de México, Dirección General de Asuntos del Personal Académico (DGAPA) project PAPIIT IN 229807 and by CONACyT project No. 2004-CO1-47339/A-1. Thanks and appreciation is due to Alfonso N. García Aldrete, Héctor M. Hernández Macías, Fernando Álvarez, František Moravec, Delane Kritsky, Héctor Espinosa Pérez, and Edgar F. Mendoza Franco for discussion of the manuscript. Thanks are also due to Felipe Villegas Márquez who prepared the illustration. I appreciate the helpful suggestions of two anonymous reviewers and of Dr. Norman Dronen that very much contribute to improve the manuscript. 


\section{References}

Aguilar-Aguilar, R., Contreras-Medina, R. \& Salgado-Maldonado, G. (2003) Parsimony Analysis of Endemicity (PAE) of Mexican hydrological basins based on helminth parasites of freshwater fishes. Journal of Biogeography, 30, 1861-1872.

Aguilar-Aguilar, R., Salgado-Maldonado, G., Contreras-Medina, R. \& Martínez-Aquino, A. (2008) Richness and endemism of helminth parasites of freshwater fishes in Mexico. Biological Journal of the Linnean Society, 94, $435-444$.

Aguirre-Macedo, M. L. \& Scholz, T. (2005) Culuwiya cichlidorum n. sp. (Digenea: Haploporidae) from the black-belt cichlid Vieja maculicauda (Pisces: Cichlidae) from Nicaragua. Journal of Parasitology, 91, 1379-1384.

Aguirre-Macedo, M. L., Scholz, T., González-Solís, D., Vidal-Martínez, V. M., Posel, P., Arjona-Torres, G., Siu-Estrada, E. \& Dumailo, S. (2001a) Larval helminths parasitizing freshwater fishes from the Atlantic coast of Nicaragua. Comparative Parasitology, 68, 42-51.

Aguirre-Macedo, M. L., Scholz, T., González-Solís, D., Vidal-Martínez, V. M., Posel, P., Arjona-Torres, G.,Dumailo, S. \& Siu-Estrada, E. (2001b) Some adult endohelminths parasitizing freshwater fishes from the Atlantic drainages of Nicaragua. Comparative Parasitology, 68, 190-195.

Anderson, R. C. (2000) Nematode Parasites of Vertebrates. CABI Publishing, Oxon 650 pp.

Bãnãrescu, P. 1995. Zoogeography of Fresh Waters. Volume 3. AULA- Verlag Wiesbaden. 1099-1617.

Bentz, S., Sinnppah-Kang, N. D., Lee-Hong, S. L., Boris, L., Combes, C. \& Verneau, O. (2006) Historical biogeography of amphibian parasites, genus Polystoma (Monogenea: Polystomatidae). Journal of Biogeography, 33, 742-749.

Bermingham, E. \& Martin, A. P. (1998) Comparative mtDNA phylogeography of neotropical freshwater fishes: testing shared history to infer the evolutionary landscape of lower Central America. Molecular Ecology, 7, 499-517.

Bravo-Hollis, M. \& Arroyo, G. (1962) Tremátodos de peces de Costa Rica I. Sobre dos especies del género Crassicutis Manter, 1936 (Lepocreadiidae Nicoll, 1914) del intestino de Cichlasoma sp. Revista de Biología Tropical, 10, 229235.

Brenes, R. R. (1961) Catálogo de los helmintos parásitos de Costa Rica. Revista de Biología Tropical, 91, 67-95.

Briggs, J. C. (1995) Global Biogeography. Elsevier. Amsterdam. 452 pp.

Bussing, W. A. (1976) Geographic distribution of the San Juan Ichthyofauna of Central America with remarks on its origin and ecology. In Investigations of the Ichthyofauna of Nicaraguan Lakes, Thorson, T. R. (ed.). School of Life Sciences, University of Nebraska-Lincoln, p. 157-175.

Bussing, W. A. (1985) Patterns of distribution of the Central American ichthyofauna. In The great American Biotic Interchange, Stehli, F. G. \& Webb, S. D. (eds.). Plenum Press, New York, p. 453-473.

Bussing, W. A. (1998) Peces de las aguas continentales de Costa Rica. Revista de Biología Tropical, 46, Suplemento 2,1468.

Caballero y C. E. \& Brenes, R. R. (1957) Helmintos de la República de Costa Rica VI. Algunos tremátodos de peces, reptiles y mamíferos. Anales del Insituto de Biología Universidad Nacional Autónoma de México, 28, 217-240.

Cabañas-Carranza, G. \& Caspeta-Mandujano, J. M. (2007) A new cucullanid species (Nematoda) from the freshwater fish Vieja intermedia (Günther, 1862) (Cichlidae) in Mexico. Journal of Parasitology, 93, 643-649.

Chambrier, A. de, \& Vaucher, C. (1984) Proteocephalus gaspari n. sp. (Cestoda: Proteocephalidae), parasite de Lepisosteus tropicus (Gill) au Lac Managua (Nicaragua). Revue Suisse de Zoologie, 91, 229-233.

Choudhury, A., Hartvigsen, R. \& Brooks, D. R. (2002) Wallinia chavarriae n. sp. (Trematoda: Macroderoididae) in Astyanax aeneus (Günther, 1860) and Bryconamericus scleroparius (Regan, 1908) (Osteichthyes: Characidae) from the Área de Conservación Guanacaste, Costa Rica. Journal of Parasitology, 88, 107-112.

Chubb, J. C. (1963) On the characterization of the parasite fauna of the fish of Llyn Tegid. Proceedings of the Zoological Society of London, 141, 609-621.

Coates, A. G. \& Obando, J. A. (1996) The geologic evolution of the Central American Isthmus, In Evolution and Environment in Tropical America, Jackson, B. C. J., Budd, A. F. \& Coates, A. G. (eds.). The University of Chicago Press, Chicago p. 21-56.

Ditrich, O., Scholz, T., Aguirre-Macedo, M. L. \& Vargas-Vázquez, J. (1997) Larval stages of trematodes from freswater molluscs of the Yucatan Peninsula, Mexico. Folia Parasitologica, 44, 109-127.

Ditrich, O., Scholz, T. \& Vargas-Vázquez, J. (1996) Life-cycle of Echinochasmus macrocaudatus n. sp. (Trematoda: Echinostomatidae). Systematic Parasitology, 33, 225-235.

Dogiel, V. A. (1961) Ecology of Parasites of Freshwater Fish, In Parasitology of Fishes, Dogiel V. A., Petrushevski, G. K. \& Polyanski, Yu (eds.). (English Traslation) Edinburgh Oliver \& Boyd, pp. 1-47.

Donnelly, T. W. (1989) Geologic history of the Caribbean and Central America, In The Geology of North America - An overview, Bally, A. W. \& Palmer, A. R. (eds.). Colorado Geological Society of America, pp. 299-321.

Froese, R. \& Pauly, D. (eds.). (2005) FishBase World Wide Web electronic publication. Available from: www.fishbase.org, version accessed May 2008.

González-Solís, D. \& Moravec, F. (2004) Two new nematode species, Orientatractis campechensis n. sp. and Orien- 
tatractis chiapasensis n. sp. (Nematoda: Atractidae) from cichlid fishes in southern Mexico and Nicaragua. Journal of Parasitology, 90, 1443-1449.

González-Solís, D., Moravec, F. \& Vidal-Martínez, V. M. (2002) Procamallanus (Spirocamallanus) chetumalensis n. sp. (Nematoda: Camallanidae) from the mayan sea catfish, Ariopsis assimilis, off the caribbean coast of Mexico. Journal of Parasitology, 88, 765-768.

Hall, C. \& Pérez-Brignoli, H. (2003) Historical Atlas of Central America. University of Oklahoma Press, Normann.

Hanek, G. \& Fernando, C. H. (1972) Monogenetic trematodes from New Providence Island, Bahamas. Journal of Parasitology, 58, 1117-1118.

Hanek, G., Molnar, K., \& Fernando, C. H. (1974) Three new genera of Dactylogyridae (Monogenea) from freshwater fishes of Trinidad. Journal of Parasitology, 60, 911-913.

Harris, P. D. (1986) Species of Gyrodactylus von Nordmann, 1832 (Monogenea Gyrodactylidae) from poeciliid fishes, with a description of G. turnbulli sp. n. from the guppy, Poecilia reticulata Peters. Journal of Natural History, 20, 183-191.

Harris, P. D. \& Lyles, A. M. (1992) Infections of Gyrodactylus bullatarudis and Gyrodactylus turnbulli on guppies (Poecilia reticulata) in Trinidad. Journal of Parasitology, 78, 912-914.

Kohn, A., Cohen, S. C. \& Salgado-Maldonado, G. (2006) Checklist of Monogenea parasites of freshwater and marine fishes, amphibians and reptiles from Mexico, Central America and Caribbean. Zootaxa, 1289, 1-114.

Kritsky, D. C. \& Fritts, T. H. (1970) Monogenetic trematodes from Costa Rica with the proposal of Anacanthocotyle gen. nov. (Gyrodactylidae: Isancistrinae). Proceedings of the Helminthological Society of Washington, 37, 63-68.

Kritsky, D. C. \& Leiby, P. D. (1972) Dactylogyridae (Monogenea) from the freshwater fish, Astyanax fasciatus (Cuvier), in Costa Rica, with descriptions of Jainus hexops sp. n., Urocleidoides costaricensis, and U. heteroancistrium combs. n. Proceedings of the Helminthological Society of Washington, 39, 227-230.

Kritsky, D. C. \& Mendoza-Franco, E. (2003) Neotropical Monogenoidea 42. Pavanelliella scaphiocotylus sp. nov. (Dactylogyridae) from the nasal cavity of the Guatemalan Chulin, Rhamdia guatemalensis (Siluriformes: Heptapteridae), from a cenote on the Yucatán Peninsula, Mexico. Comparative Parasitology, 70, 136-139.

Kritsky, D.C., Mendoza-Franco, E. F. \& Scholz, T. (2000) Neotropical Monogenoidea. 36. Dactylogyrids from the gills of Rhamdia guatemalensis (Siluriformes: Pimelodidae) from cenotes of the Yucatan Peninsula, Mexico, with proposal of Ameloblastella gen. n. and Aphanoblastella gen. n. (Dactylogyridae: Ancyrocephalinae). Comparative Parasitology, 67, 76-84.

Kritsky, D. C. \& Thatcher, V. E. (1974) Monogenetic trematodes (Monopisthocotylea: Dactylogyridae) from freshwater fishes of Colombia, South America. Journal of Helminthology, 48, 59-66.

Kritsky, D. C., Thatcher, V. E. \& Boeger, W. A. (1986) Neotropical Monogenea. 8. Revision of Urocleidoides (Dactylogyridae, Ancyrocephalinae). Proceedings of the Helminthological Society of Washington, 53, 1-37.

Kritsky, D. C., Thatcher, V. E. \& Boeger, W. A. (1989) Neotropical Monogenea. 15. Dactylogyrids from the gills of Brazilian Cichlidae with proposal of Sciadicleithrum gen. n. (Dactylogyridae). Proceedings of the Helminthological Society of Washington, 56, 128-140.

Kritsky, D.C., Vidal-Martínez, V. M. \& Rodríguez-Canul, R. (1994) Neotropical Monogenoidea 19. Dactylogyridae of cichlids (Perciformes) from the Yucatán Peninsula, with descriptions of three new species of Sciadicleithrum Kritsky, Thatcher, and Boeger, 1989. Journal of the Helminthological Society of Washington, 61, 26-33.

Lamothe-Argumedo, R. (1977) Caballeroiella isabellae gen. nov. sp. nov. (Trematoda: Hemiuridae) parásito de Rhamdia guatemalensis, In Excerta Parasitologica en memoria del Dr. Eduardo Caballero y Caballero. Publicación especial número 4, Instituto de Biología, Universidad Nacional Autónoma de México, México, D. F., pp. $207-213$.

Lamothe-Argumedo, R. \& Ponciano-Rodríguez, G. (1986a) Revisión de la Subfamilia Acanthostominae Nicoll, 1914 y establecimiento de dos nuevos géneros. Anales del Instituto de Biología, Universidad Nacional Autónoma de México, Serie Zoología, 56, 301-322.

Lamothe-Argumedo, R. \& Ponciano-Rodríguez, G. (1986b) Estudio helmintológico de los animales silvestres de la Estación de Biología Tropical "Los Tuxtlas", Veracruz. Trematoda III. Redescripción de Stunkardiella minima (Stunkard, 1938) Lamothe y Ponciano, 1985. Anales del Instituto de Biología, Universidad Nacional Autónoma de México, Serie Zoología, 56, 323-336.

Maldonado-Koerdell, M. (1964) Geohistory and paleography of Middle America. Pp. 3-32 In Handbook of Middle American indians. Volume One. Wauchope, R. \& West, R. C. (eds.), University of Texas Press, Austin. 570 pp.

Mendoza-Franco, E. F., Aguirre-Macedo, M. L. \& Vidal-Martínez, V. M. (2007) New and previously described species of Dactylogyridae (Monogeneoidea) from the gills of Panamanian freshwater fishes (Teleostei). Journal of Parasitology, 93, 761-771.

Mendoza-Franco, E. F., Kritsky, D. C., Vidal-Martínez, V. M., Scholz, T. \& Aguirre-Macedo, M. L. (2004) Neotropical Monogenoidea. 45. Revision of Diplectanocotyla Yamaguti, 1953 (Diplectanidae) with redescription of Diplectanocotyla megalopis Rakotofiringa and Oliver, 1987 on Atlantic Tarpon, Megalops atlanticus Cuvier and Valenciennes, from Nicaragua and Mexico. Comparative Parasitology, 71, 158-165.

Mendoza-Franco, E. F., Posel, P. \& Dumalio, S. (2003a) Monogeneans (Dactylogyridae: Ancyrocephalinae) of freshwa- 
ter fishes from the Caribbean coast of Nicaragua. Comparative Parasitology, 70, 32-41.

Mendoza-Franco, E. F., Scholz, T. \& Cabañas-Carranza, G. (2003b) Guavinella tropica n. gen., n. sp. (Monogenea: Dactylogyridae) from the gills of the bigmouth sleeper, Gobiomorus dormitor (Perciformes: Eleotridae), from Mexico. Comparative Parasitology, 70, 26-31.

Mendoza-Franco, E. F., Scholz, T. \& Vidal-Martínez, V. M. (1997) Sciadicleithrum meekii sp. n. (Monogenea: Ancyrocephalinae) from the gills of Cichlasoma meeki (Pisces: Cichlidae) from cenotes (= sinkholes) of the Yucatan Peninsula, Mexico. Folia Parasitologica, 44, 205-208.

Mendoza-Franco, E. F., Scholz, T., Vivas-Rodríguez, C. \& Vargas-Vázquez, J. (1999) Monogeneans of freshwater fishes from cenotes (sinkholes) of the Yucatan Peninsula, Mexico. Folia Parasitologica, 46, 267-273.

Mendoza-Franco, E. F. \& Vidal-Martínez, V. M. (2001) Salsuginus neotropicalis n. sp. (Monogenea: Ancyrocephalinae) from the pike killifish Belonesox belizanus (Atheriniformes: Poeciliidae) from southeastern Mexico. Systematic Parasitology, 48, 41-45.

Mendoza-Franco, E., Vidal-Martínez, V., Aguirre-Macedo, L., Rodríguez-Canul, R. \& Scholz, T. (2000) Species of Sciadicleithrum (Dactylogyridae: Ancyrocephalinae) of cichlid fishes from southeastern Mexico and Guatemala: new morphological data and host and geographical records. Comparative Parasitology, 67, 85-91.

Mendoza-Franco, E. F., Vidal-Martínez, V. M., Cruz-Quintana, Y. \& Prats-León, F. L. (2006) Monogeneans on native and introduced freshwater fishes from Cuba with the description of a new species of Salsuginus Beverley-Burton, 1984 from Limia vittata (Poeciliidae). Systematic Parasitology, 64, 181-190.

Miller, R. R. (1966) Geographical distribution of Central American freshwater fishes. Copeia 1966: 773-802.

Miller, R. R., Minckley, W. L. \& Norris, S. M. (2005) Freshwater Fishes of México. The University of Chicago Press. $490 \mathrm{pp}$.

Miller, R. R. \& Norris, S. M. (2005) Family Cichlidae pp. 352-386 In Freshwater Fishes of México, Miller, R. R., Minckley, W. L. \& Norris, S. M. (eds.). The University of Chicago Press. 490 pp.

Minckley, W. L., Miller, R. R., Barbour, C. D., Schmitter-Soto, J. J. \& Norris, S. M. (2005) Historical Ichthyogeography pp. 24-47 In Freshwater Fishes of México, Miller, R. R., Minckley, W. L. \& Norris, S. M. (eds). The University of Chicago Press. 490 pp.

Mizelle, J. D. \& Kristky, D. C. (1969) Studies on monogenetic trematodes XXXIX. Exotic species of Monopisthocotylea with the proposal of Archidiplectanum gen. n. and Longihaptor gen. n. American Midland Naturalist, 81, 370-386.

Mizelle, J. D. \& Price, C. E. (1964) Studies on monogenetic trematodes XXVII. Dactylogyrid species with the proposal of Urocleidoides gen. n. Journal of Parasitology, 60, 914-920.

Molnar, K., Hanek, G. \& Fernando, C. H. (1974) Ancyrocephalids (Monogenea) from freshwater fishes of Trinidad. Journal of Parasitology, 60, 914-920.

Moravec, F. (1994) Parasitic nematodes of freshwater fishes of Europe. Academia Praha 473 pp.

Moravec, F. (1998) Nematodes of freshwater fishes of the Neotropical Region. Academia Praha, 464 pp.

Moravec, F. (2000) Nematodes as parasites of inland fishes in Mexico pp. 155-165 In Metazoan parasites in the neotropics: a systematic and ecological perspective, Salgado-Maldonado, G., García Aldrete, A. N. \& Vidal-Martínez, V. M. (eds.), Instituto de Biología Universidad Nacional Autónoma de México.

Moravec, F. \& Coy-Otero, A. (1987) Rhabdochona cubensis sp. n. (Nematoda: Rhabdochonidae) from the freshwater fish Gambusia punctata from Cuba. Helminthologia, 24, 103-110.

Moravec, F., Mendoza-Franco, E., Vargas-Vázquez, J. \& Vivas-Rodríguez, C. (1995a) Studies on the development of Procamallanus (Spirocamallanus) rebecae (Nematoda: Camallanidae), a parasite of cichlid fishes in Mexico. Folia Parasitologica, 42, 281-292.

Moravec, F. \& Huffman, D. G. (2001) Observations on the biology of Rhabdochona kidderi texensis, a parasite of North American cichlids. Journal of Helminthology, 75, 197-203.

Moravec, F. \& Salgado-Maldonado, G. (2002) Redescription of Perezitrema bychowskyi (Caballero \& Caballero, 1975) (Trematoda: Macroderoididae), with remarks on the systematic status of Perezitrema Baruš \& Moravec, 1996. Systematic Parasitology, 53, 199-206.

Moravec, F., Salgado-Maldonado, G. \& Aguilar-Aguilar, R. (2002) Neophilometroides n. gen. (Nematoda: Philometridae) for Philometroides caudatus Moravec, Scholz and Vivas-Rodríguez, 1995, with erection of Neophilometroidinae n. subfam. Journal of Parasitology, 88, 774-777.

Moravec, F. \& Vargas-Vázquez, J. (1996) The development of Procamallanus (Spirocamallanus) neocaballeroi (Nematoda: Camallanidae), a parasite of Astyanax fasciatus (Pisces) in Mexico. Folia Parasitologica, 43, 61-70.

Moravec, F., Vargas-Vázquez, J. \& González-Solís, D. (1996) Nematode parasites from the blind fish Ogilbia pearsei from the Nohoch cave system with remarks on Rhabdochona kidderi (Nematoda) from fishes of Yucatán, Mexico, Acta Societatis Zoologicae Bohemicae, 63, 295-300.

Moravec, F., Vivas-Rodríguez, C., Scholz, T., Vargas-Vázquez, J., Mendoza-Franco, E. \& González-Solís, D. (1995b) Nematodes parasitic in fishes of cenotes (= sinkholes) of the Peninsula of Yucatan, Mexico. Part 1. Adults. Folia Parasitologica, 42, 115-129.

Myers, G. S. (1938) Fresh-water fishes and West Indian zoogeography. Annual Reports Smithsonian Institution, 1937, 
339-364.

Myers, G. S. (1966) Derivation of the freshwater fish fauna of central America. Copeia, 1966, 766-773.

Nelson, J. S. (2006) Fishes of the world. Fourth edition. John Wiley and Sons, Inc.

Pearse, A. S. (1936) Parasites from Yucatan. In: Pearse, A. S., Creaser, E. P. \& Hall, F. G. (Eds.), The cenotes of Yucatan: a zoological and hydrographic survey. Washington, Carnegie Institution, Publication 457, 45-59.

Pérez, G. \& Choudhury, A. (2005) Biogeography of helminth parasites of freshwater fishes in Mexico: the search for patterns and processes. Journal of Biogeography, 32, 645-659.

Petter, A. J. (1977) Description dún nouveau nématode parasite de poisson, en Guadeloupe: Cucullanus caballeroi n. sp. Excerta Parasitológica en Memoria del Dr. eduardo Caballero y Caballero. Instituto de Biología, Universidad Nacional Autónoma de México, Publicaciones especiales 4: 459-462.

Petter, A. J., Golvan, Y. J. \& Tcheprakoff, R. (1977) Nématodes de poissons de rivière en Guadeloupe. Bulletin du Muséum National d'Histoire Naturelle, Paris, $3^{e}$ série Zoologie, 298, 159-171.

Price, C.E. (1967) Dactylogyrus mizellei, a new monogentic trematode from Latin America. Annales de Zoologie Agra, $5,65-69$.

Price, C. E. \& Bussing, W. A. (1967) Monogenean parasites of Costa Rican fishes. Part 1. Descriptions of two new species of Cleiodiscus Müller, 1934. Rivista di Parassitologia, 28, 81-86.

Price, C. E. \& Bussing, W. A. (1968) Monogenean parasites of Costa Rican fishes. II. Proposal of Palombitrema heteroancistrium n. gen., n. sp. Proceedings of the Helminthological Society of Washington, 35, 54-57.

Price, E. W. (1938) The Monogenetic trematodes of Latin America. Livro Juvilar do Professor Lauro Travassos. Rio de Janeiro, Brazil 407-413.

Rand, T. G. \& Wiles, M. (1987). Salsuginus bermudae sp. n. (Monogenea: Ancyrocephalidae) from Fundulus bermudae Gunther and Gambusia affinis (Baird and Girard) in Bermuda. Canadian Journal of Zoology, 65, 1847-1852.

Roe, K. J., Conkel, D. \& Lydeard, C. (1997) Molecular systematic of Middle American cichlid fishes and the evolution of trophic-types in "Cichlasoma (Amphilophus)" and "C. (Thorichthys)". Molecular Phylogenetics and evolution, 7 , 366-376.

Rosas-Valdez, R., Choudhury, A. \& Pérez, G. (2004) Phyllogenetic analysis of genera of Corallobothriinae (Cestoda: Proteocephalidea) from North American ictalurid fishes, using partial sequences of the $28 \mathrm{~S}$ ribosomal gene. Journal of Parasitology, 90, 1123-1127.

Rosen, D. E. (1974) Phylogeny and zoogeography of salmoniform fishes and relationships of Lepidogalaxias salamandroides. Bulletin of the American Museum of Natural History, 153, 265-326.

Rosen, D. E. (1976) A vicariance model of Caribbean biogeography. Systematic Zoology, 24, 431-464.

Rosen, D. E. (1985) Geological hierarchies and biogeographic congruence in the Caribbean. Annals of the Missouri Botanical Garden, 72, 636-659.

Salgado-Maldonado, G. (1978) Acantocéfalos de peces IV. Descripción de dos especies nuevas de Neoechinorhynchus Hamann, 1892 (Acanthocephala: Neoechinorhynchidae) y algunas consideraciones sobre este género. Anales del Instituto de Biología, Universidad Nacional Autónoma de México, Serie Zoología, 49, 35-48.

Salgado-Maldonado, G. (1982) Acanthocephala pp. 121-131 In Aquatic Biota of Mexico, Central America and the West Indies, Hurlbert, S.H. \& Villalobos-Figueroa, A. (eds.), San Diego California, San Diego State University.

Salgado-Maldonado, G. (2006) Checklist of helminth parasites of freshwater fishes from Mexico. Zootaxa, $1324,1-357$.

Salgado-Maldonado, G., Aguilar-Aguilar, R., Cabañas-Carranza, G., Soto-Galera, E. \& Mendoza-Palmero, C. (2005) Helminth parasites in freshwater fish from the Papaloapan river basin, Mexico. Parasitology Research, 96, 69-89.

Salgado-Maldonado, G., Cabañas-Carranza, G., Caspeta-Mandujano, J. M., Soto-Galera, E., Mayén-Peña, E., Brailovsky, D. \& Baéz-Valé, R. (2001) Helminth parasites of freswater fishes of the Balsas River drainage, Southwestern Mexico. Comparative Parasitology, 68, 196-203.

Salgado-Maldonado, G., Cabañas-Carranza, G., Soto-Galera, E., Pineda-López, R., Caspeta-Mandujano, J. M., AguilarCastellanos, E. \& Mercado-Silva, N. (2004a) Helminth parasites of freshwater fishes of the Pánuco river basin, East Central Mexico. Comparative Parasitology, 71, 190-202.

Salgado-Maldonado, G., Jiménez-García, M. I. \& León-Règagnon, V. (1992) Presence of Octospiniferoides chandleri Bullock, 1957 in Heterandria bimaculata from Catemaco, Veracruz, and considerations about the acanthocephalans of fresh water fishes of Mexico. Memorias do Instituto Oswaldo Cruz, 87 Suppl. I, 239-240.

Salgado-Maldonado, G., Mercado-Silva, N., Cabañas-Carranza, G., Caspeta-Mandujano, J. M., Aguilar-Aguilar, R. \& Íniguez-Dávalos, L. I. (2004b) Helminth parasites of freshwater fishes of the Ayuquila river, Sierra de Manantlán Biosphere Reserve, west central Mexico. Comparative Parasitology, 71, 67-72.

Savage, J. M. (1966) The origins and history of the Central American herpetofauna. Copeia , 1966, 719-766.

Scholz, T., Aguirre-Macedo, M. L. \& Choudhury, A. (2004) Auriculostoma astyanace n. gen., n. sp. (Digenea: Allocreadiidae), from the Banded Astyanax, Astyanax fasciatus (Characiformes: Characidae), from Nicaragua, with a reevaluation of Neotropical Crepidostomum spp. Journal of Parasitology, 90, 1128-1132.

Scholz, T., Ditrich, O. \& Vargas-Vázquez, J. (1996a) Echinochasmus leopoldinae n. sp. (Trematoda: Echinostomatidae) and data on its life-cycle. Systematic Parasitology, 33, 157-165. 
Scholz, T., Lavadores, I. P., Vargas, J., Mendoza, F. E., Rodríguez, R. \& Vivás, C. (1994). Life cycle of Oligogonotylus manteri (Digenea: Cryptogonimidae), a parasite of cichlid fishes in southern Mexico. Journal of the Helminthological Society of Washington, 61, 190-199.

Scholz, T., Pech-Ek, M. C. F. \& Rodríguez-Canul, R. (1995a) Biology of Crassicutis cichlasomae, a parasite of cichlid fishes in Mexico and Central America. Journal of Helminthology, 69, 69-75.

Scholz, T. \& Salgado-Maldonado, G. (1994) On Genarchella isabellae (Digenea: Derogenidae) from cichlid and pimelodid fishes in Mexico. Journal of Parasitology, 80, 1013-1017.

Scholz, T., Vargas-Vázquez, J. \& Moravec, F. (1996b) Bothriocephalus pearsei n. sp. (Cetoda: Psudophyllidea) from cenote fishes of the Yucatan Peninsula, Mexico. Journal of Parasitology, 82, 801-805.

Scholz, T., Vargas-Vázquez, J., Moravec, F., Vivás-Rodríguez, C. \& Mendoza-Franco, E. (1996c) Cestoda and Acanthocephala of fish from cenotes (= sinkholes) of Yucatan, Mexico. Folia Parasitologica, 43, 141-152.

Scholz, T., Vargas-Vázquez, J. \& Salgado-Maldonado, G. (1995b) Revision of Genarchella species (Digenea: Derogenidae) parasitizing freshwater fishes in Mexico and Central America. Journal of Natural History, 29, $1403-1417$.

Smith, D. L. (1985) Caribbean plate relative motions, pp. 17-48, In The Great American Interchange, F. G. Stehli, \& Webb, S. D. (eds.). Plenum Press New York.

Sparks, J. S. \& Smith, W. L. (2005) Freshwater fishes, dispersal ability, and nonevidence: "Gondwana Life Rafts" to the rescue. Systematic Biology, 54, 158-165.

Stehli, F. G. \& Webb, S. D. (1985) A kaleidoscope of plates, faunal and floral dispersal, and sea level changes, pp. 3-16 In The Great American Interchange, F. G. Stehli, \& Webb, S. D. (eds.). Plenum Press New York.

Stuart, L. C. (1964) Fauna of Middle America pp. 316-362 In Handbook of Middle American indians. Volume One, Wauchope, R. \& West, R. C. (eds). University of Texas Press, Austin. 570 pp.

Stuart, L. C. (1966) The environment of the Central American cold-blooded vertebrate fauna. Copeia, 1966, 684-699.

Szidat, L. (1954) Tremátodos nuevos de peces de agua dulce de la República Argentina y un intento para aclarar su carácter marino. Revista del Instituto Nacional de Investigaciones de Ciencias Naturales Buenos Aires, 3, 1-85.

Tamayo, J. \& West, R. C. (1964) The hydrography of Middle America. pp. 84-121 In Handbook of Middle American indians. Volume One, Wauchope, R. \& West, R. C. (eds), University of Texas Press, Austin. 570 pp.

Thatcher, V. E. (2006) Amazon Fish Parasites. Aquatic Biodiversity in Latin America. Pensoft. Sofia. 508 pp.

Turnbull, E. R. (1956) Gyrodactylus bullatarudis n. sp. from Lebistes reticulatus Peters with a study of its life cycle. Canadian Journal of Zoology, 34, 583-594.

Vidal-Martínez, V. M., Aguirre-Macedo, M. L., Scholz, T., González-Solís, D. \& Mendoza-Franco, E. F. (2001a) Atlas of the helminth parasites of cichlid fish of Mexico. Academia, Praha pp. 165.

Vidal-Martínez, V.M. \& Kennedy, C. R. (2000) Zoogeographic determinants of the composition of the helminth fauna of neotropical cichlid fish, pp. 227-290 In Metazoan parasites in the Neotropics: a systematic and ecological perspective, Salgado-Maldonado, G., García Aldrete, A. N. \& Vidal-Martínez, V. M. (eds.). Instituto de Biología Universidad Nacional Autónoma de México.

Vidal-Martínez, V.M., Scholz, T. \& Aguirre-Macedo, M. L. (2001b) Dactylogyridae of cichlid fishes from Nicaragua, Central America, with descriptions of Gussevia herotilapiae sp. n. and three new species of Sciadicleithrum (Monogenea: Ancyrocephalinae). Comparative Parasitology, 68, 76-86.

Vinjoy, M., Prieto, A., Fajer, E. \& Cartaya, E. (1985) Presencia de Crassicutis sp. (Trematoda: Allocreadidae) en el tracto intestinal de la biajaca (Cichlasoma tetracanthum) en Cuba. Revista de Salud Animal, 7, 287-290.

Watson, D. E. (1976) Digenae of fishes from Lake Nicaragua, pp. 251-260 In Investigations of the Ichthyofauna of Nicaraguan Lakes, Thorson, T. R. (ed.), School of Life Sciences, University of Nebraska-Lincoln.

Watson, D. E. \& Thorson, T. R. (1976) Helminths from elasmobranchs in Central American freshwaters, pp. 629-640 In Investigations of the Ichthyofauna of Nicaraguan Lakes, Thorson, T. R. (ed.), School of Life Sciences, University of Nebraska-Lincoln.

Wootten, R. (1973) The metazoan parasite-fauna of fish from Hanningfield Reservoir, Essex in relation to features of the habitat and host populations. Journal Zoology London, 171, 323-331. 